\title{
Boundary State from Ellwood Invariants
}

\author{
Matěj Kudrna $^{(a)}$, Carlo Maccaferri ${ }^{(a, b)}$ 2, Martin Schnabl ${ }^{(a)} \sqrt{3}$ \\ (a) Institute of Physics of the ASCR, v.v.i. \\ Na Slovance 2, 182 21 Prague 8, Czech Republic \\ (b) Dipartimento di Fisica, Universitá di Torino and INFN Sezione di Torino \\ Via Pietro Giuria 1, I-10125 Torino, Italy
}

\begin{abstract}
Boundary states are given by appropriate linear combinations of Ishibashi states. Starting from any open string field theory solution and assuming Ellwood conjecture we show that every coefficient of such a linear combination is given by an Ellwood invariant, computed in a slightly modified theory where it does not trivially vanish by the on-shell condition. Unlike the previous construction of Kiermaier, Okawa and Zwiebach, ours is linear in the string field, it is manifestly gauge invariant and it is also suitable for solutions known only numerically. The correct boundary state is readily reproduced in the case of known analytic solutions and, as an example, we compute the energy momentum tensor of the rolling tachyon from the generalized invariants of the corresponding solution. We also compute the energy density profile of Siegel-gauge multiple lump solutions and show that, as the level increases, it correctly approaches a sum of delta functions. This provides a gauge invariant way of computing the separations between the lower dimensional D-branes.
\end{abstract}

\footnotetext{
${ }^{1}$ Email: matej.kudrna at email.cz

${ }^{2}$ Email: maccafer at gmail.com

${ }^{3}$ Email: schnabl.martin at gmail.com
} 


\section{Contents}

1 Introduction 3

2 Boundary state from Ellwood invariants 6

2.1 Generalizing the Ellwood invariant . . . . . . . . . . . . . . . 6

2.1.1 Lifting solutions . . . . . . . . . . . . . . . . . . . . 7

2.1.2 Lifting closed string states . . . . . . . . . . . . . . . . 11

2.1.3 Generalized Ellwood invariant . . . . . . . . . . . . . . . 12

2.2 Ellwood invariants and Ishibashi states . . . . . . . . . . . . 13

2.3 Ellwood invariants and boundary primaries . . . . . . . . . . . 17

3 Analytic solutions: Rolling tachyon 20

4 Numerical solutions: Lumps in Siegel gauge 27

4.1 Moeller-Sen-Zwiebach lump at $R=\sqrt{3} \ldots \ldots \ldots \ldots$. . . . . . . . . .

4.2 Double lumps at $R=2 \sqrt{3} \ldots \ldots \ldots \ldots \ldots \ldots$. . . . . . . . . . . . . .

5 Conclusions 39

A Example of BCFT ${ }^{\text {aux }} 41$

B Conservation laws for the Ellwood invariant 42

B.1 Review of conservation laws of the identity string field . . . . . . . . 43

B.2 Virasoro conservation laws . . . . . . . . . . . . . . . . . . 43

B.3 Oscillator conservation laws . . . . . . . . . . . . . . 46

B.4 Ghost conservation laws . . . . . . . . . . . . . . . . . . . 47

C General properties of the boundary state 49

C.1 Proof of matter ghost factorization . . . . . . . . . . . . . . 50

C.2 Normalization of the ghost boundary state . . . . . . . . . . . 51

D Some more lumps 


\section{Introduction}

In attempts to explore the landscape of open string field theory [1] either by analytic or numerical means, one faces the problem of a physical identification of the solutions to the equation of motion. They are believed to be in one-to-one correspondence with allowed boundary states for given bulk CFT, but so far we have had only limited tools to identify the respective boundary state [4, 5, 6]. In [6] a geometric construction of the boundary state was given, in principle, for any classical solution of open string field theory (OSFT). However, due to the nonlinearity of the construction, it is not known how to explicitly perform computations for generic solutions like, for example, the important class of Siegel-gauge level truncated solutions. Moreover, the OSFT boundary state of [6] is not guaranteed to be gauge invariant, and the BCFT boundary state is recovered only up to BRST-exact terms (which are however absent in several explicit examples of wedge-based analytic solutions).

In this work we present a remarkably simple method to construct explicitly, in a gauge invariant way, the BCFT boundary state from a given solution. The main advantage of the method is that, while it easily gives the expected results for known analytic solutions, it also works reasonably well for solutions known only numerically. The key ingredient of our construction is the widely believed, but as yet unproven Ellwood's conjecture [5, 6], which can be simply re-stated as

$$
\left\langle\mathcal{V}_{c l}\left|c_{0}^{-}\right| B_{\Psi}\right\rangle=-4 \pi i\left\langle I\left|\mathcal{V}_{c l}(i)\right| \Psi-\Psi_{\mathrm{TV}}\right\rangle
$$

where $\mathcal{V}_{c l}$ is an on-shell closed string vertex operator of ghost number two, $\Psi$ is a solution of the OSFT, $\left|B_{\Psi}\right\rangle$ is the corresponding boundary state, and finally $\Psi_{\mathrm{TV}}$ is the tachyon vacuum (in any gauge or form). Note that the left hand side is evaluated using the closed string inner product, while the right hand is evaluated using the open string inner product.

This equation, however, constrains only the tiny on-shell part of the boundary state. For example, for spatially constant string fields the only nontrivial component of the corresponding boundary state which can be computed this way is just the zero momentum massless closed string mode with vertex operator of the form $\xi_{\mu \nu} \partial X^{\mu} \bar{\partial} X^{\nu}$. A possible way around this problem has in fact been hinted at already in [7], before the Ellwood's conjecture had been formulated. The trick is essentially to assume the existence of some spacetime direction with Dirichlet boundary condition. The vertex operator can then be taken to have arbitrary momentum dependence in the directions we are interested in. To put the whole operator on-shell, we adjust the momentum of the closed string vertex operator in the extra direction with Dirichlet boundary condition. Due to the Dirichlet condition the invariant will not vanish trivially. In this way, we can unambiguously

\footnotetext{
${ }^{1}$ For recent reviews see e.g. [2, 3].
} 
extract the overlap between the boundary state and closed string matter primaries. The goal of this paper is to make this idea more precise and to illustrate it on the examples of the analytic rolling tachyon and numerical lump solutions describing lower dimensional D-branes.

In the matter CFT, the knowledge of the inner product of the boundary state with any primary state is sufficient to determine the complete boundary state with the help of the Virasoro gluing conditions $\left(L_{n}-\bar{L}_{-n}\right)|B\rangle=0$. These conditions are solved in full generality by the conformal Ishibashi states [8], while the Ellwood invariants determine their exact linear combination. In principle, OSFT thus solves the outstanding unsolved problem of boundary conformal field theory: determine the set of all boundary conditions consistent with conformal symmetry in a given CFT. In the string theory language this is the problem of classification of all allowed D-branes in a given background. The coefficients of the Ishibashi states must satisfy lots of constraints, either from modular invariance or the so called sewing conditions. A lot of progress has been achieved in CFT attempting to solve these constraints, but much more remains. String field theory solutions, on the other hand, should provide automatically a solution to all these constraints.

Let us describe the computation of the primary components of the boundary state in a little bit more detail. The state space of open string field theory is given by the Hilbert space of the boundary conformal field theory $\mathrm{BCFT}_{0}$. Any such element in the ghost number one sector can be written as [9, 10]

$$
\Psi=\sum_{j} \sum_{\substack{I=\left\{n_{1}, n_{2}, \ldots\right\} \\ J=\left\{m_{1}, m_{2}, \ldots\right\}}} a_{I J}^{j} L_{-I}^{\text {matter }}\left|\phi_{j}\right\rangle \otimes L_{-J}^{\text {ghost }} c_{1}|0\rangle,
$$

where the index $j$ runs over all matter primaries that are 'turned on', while the multiindices $I$ and $J$ give its descendants. The tachyon vacuum does not turn on any primary other than the identity operator, while for example for the lumps an infinite number of $e^{i k X}$ primaries (among others) is required.

Given a solution $\Psi$ of string field theory built upon $\mathrm{BCFT}_{0}$, one can associate to it a solution $\tilde{\Psi}$ built upon $\mathrm{BCFT}_{0} \otimes \mathrm{BCFT}^{\text {aux }}$, which only depends on $\mathrm{BCFT}^{\text {aux }}$ through Virasoro operators and where BCFT ${ }^{\text {aux }}$ is a (non-unitary) BCFT of central charge $c=0$. In simple cases (in fact in all encountered cases) this is easily done by appropriately replacing the matter energy momentum tensor $T^{\text {matter }}$ with $T^{\text {matter }}+T^{\text {aux }}$. Let us further assume that BCFT ${ }^{\text {aux }}$ contains a bulk primary operator of dimension $(1-h, 1-h)$ with nonvanishing disk 1-point function for every weight $(h, h)$ primary in the matter part of $\mathrm{BCFT}_{0}$. One universal option is to choose a BCFT of a free boson (let us call it $Y$ ) with Dirichlet boundary condition and consider generically non-normalizable operators $e^{2 i \sqrt{1-h} Y}$. To ensure zero central charge of BCFT ${ }^{\text {aux }}$, one should tensor this free boson theory with a non-unitary theory of negative central charge, for example a $c=-1$ linear 
dilaton theory, and supplement the closed string insertion with the appropriate weight $(0,0)$ primary $w$, to soak up the background charge.2 Since $\mathrm{BCFT}_{0}$ and $\mathrm{BCFT}^{\text {aux }}$ are completely decoupled, and the uplifted solution does not turn on any BCFT ${ }^{\text {aux }}$ primaries other than the identity, the boundary conditions of BCFT ${ }^{\text {aux }}$ should not be changed by the solution. We thus expect that the boundary state for the uplifted OSFT solution has the following factorized form

$$
\left|B_{\Psi}\right\rangle^{\mathrm{CFT}_{0} \otimes \mathrm{CFT}^{\mathrm{aux}}}=\left|B_{\Psi}\right\rangle^{\mathrm{CFT}_{0}} \otimes\left|B_{0}\right\rangle^{\mathrm{CFT} \mathrm{T}^{\mathrm{aux}}} .
$$

Assuming further that the boundary state for the solution $\Psi$ itself factorizes into matter and universal ghost parts

$$
\left|B_{\Psi}\right\rangle^{\mathrm{CFT}_{0}}=\left|B_{\Psi}\right\rangle^{\mathrm{CFT}_{0}^{\text {matter }}} \otimes\left|B_{g h}\right\rangle,
$$

and decomposing $\left|B_{\Psi}\right\rangle^{\mathrm{CFT}_{0}^{\text {matter }}}$ into the basis of Ishibashi states

$$
\left.\left|B_{\Psi}\right\rangle^{\mathrm{CFT}_{0}^{\text {matter }}}=\sum_{\alpha} n_{\Psi}^{\alpha}\left|V_{\alpha}\right\rangle\right\rangle
$$

belonging to the (product of left and right) Verma modules of the matter primary operators $V_{\alpha}$, we can determine all the coefficients from the knowledge of the generalized Ellwood invariants

$$
n_{\Psi}^{\alpha}=2 \pi i\left\langle I\left|\mathcal{V}^{\alpha}(i)\right| \tilde{\Psi}-\tilde{\Psi}_{\mathrm{TV}}\right\rangle^{\mathrm{BCFT}_{0} \otimes \mathrm{BCFT}^{\mathrm{aux}}}
$$

where

$$
\mathcal{V}^{\alpha}=c \bar{c} V^{\alpha} e^{2 i \sqrt{1-h_{\alpha}} Y} w
$$

and $V^{\alpha}$ form a dual basis in the matter part of $\mathrm{CFT}_{0}$, i.e.

$$
\left\langle V^{\alpha} \mid V_{\beta}\right\rangle=\delta_{\beta}^{\alpha} .
$$

This is our main result.

The paper is organized as follows. In section 2 we describe our construction of the boundary state in more detail. In section 3 we derive the boundary state for the rolling tachyon analytic solution, while in the subsequent section 4 we apply our construction to single and double lump numerical solutions in open string field theory. We end up with some conclusions and future perspectives. Appendix $\mathrm{A}$ contains an example of the auxiliary $c=0$ BCFT which we use to generalize the Ellwood invariants. In appendix B

\footnotetext{
${ }^{2}$ In most cases, however, such a construction is not necessary. It is enough to assume the existence of a spacetime direction along which nothing happens, and change its boundary condition to Dirichlet, if it is not Dirichlet to start with.
} 
we derive a set of conservation laws for the Ellwood invariant, which are very efficient and practical, especially in numerical computations. In appendix C we discuss various universal properties of the boundary states in bosonic string theory. In particular, we show that all conformal level-matched boundary states factorize into matter and universal ghost parts, and determine the precise form of the latter, including its normalization. Finally, appendix D contains numerical results for several Siegel-gauge lump solutions, in addition to those discussed in section 4 of the main text.

\section{Boundary state from Ellwood invariants}

In this section we construct a boundary state from a given OSFT solution in two steps. First, we generalize Ellwood conjecture in order to be able to use generic matter primaries in the Ellwood invariant. Then, we show that a generic boundary state describing conformal boundary conditions in a total matter/ghost BCFT is necessarily matter-ghost factorized, and use the Virasoro gluing condition of the matter sector to fix the nonprimary part of the matter boundary state. Finally, we comment on the relation between the boundary operators turned on by the solution, and the boundary state.

\subsection{Generalizing the Ellwood invariant}

Let $\mathrm{BCFT}_{0}$ be the reference boundary CFT on which we define OSFT. Let $\Psi$ be a solution describing another $\mathrm{BCFT}_{\Psi}$. Then Ellwood conjectured that [5, 6]

$$
-4 \pi i\langle I|\mathcal{V}(i,-i)| \Psi\rangle \equiv-4 \pi i\langle E[\mathcal{V}] \mid \Psi\rangle=\left\langle\mathcal{V}\left|c_{0}^{-}\right| B_{\Psi}\right\rangle-\left\langle\mathcal{V}\left|c_{0}^{-}\right| B_{0}\right\rangle .
$$

Here $\mathcal{V}$ is a closed string vertex operator of the form

$$
\mathcal{V}=c \bar{c} V^{\text {matter }}
$$

and

$$
\langle E[\mathcal{V}]| \equiv\langle I| \mathcal{V}(i,-i)
$$

is a corresponding state in the open string Hilbert space. Because $\mathcal{V}$ is inserted at a conical singularity (the midpoint of the identity string field) the quantity $\langle I|\mathcal{V}(i,-i)| \Psi\rangle$ is only meaningful when $\mathcal{V}$ is a weight zero primary. Luckily all the ghost-number two closed string cohomology (except for the ghost dilaton) is contained in states of this form and thus (2.1) can be used to define the on-shell part of the boundary state $\left|B_{\Psi}\right\rangle$. But this is clearly not enough to completely define the boundary state.

This is the well-known limitation of Ellwood invariants: most of the closed string tadpoles vanish by momentum conservation when the closed string is on-shell. This 
limitation lead the authors of [6] to the construction of a family of Wilson-loop-like maps from the classical solution $\Psi$ to ghost-number-three level-matched and BRST-invariant closed string states which are conjectured to be BRST equivalent to the boundary state. In particular one can probe them with off-shell closed string states. Assuming Ellwood conjecture (or alternatively, assuming background independence of a version of openclosed string field theory [6]), the BRST equivalence to the BCFT boundary state can be established. The construction is completely performed within the open string star algebra and its intrinsic nonlinearity can give nontrivial checks on the regularity of proposed OSFT classical solutions [11. But there is a simple shortcut to get precisely the BCFT-boundary state described by the classical solution $\Psi$. Suppose we are dealing with a solution which does not depend on a target space direction, say $Y$. This means that the $Y$ dependence of the solution can be taken to be universal, depending only on Virasoro generators of the $Y$-BCFT. Then the solution will remain a solution if we change the boundary conditions of the $Y$-BCFT to be Dirichlet $Y(0)=Y(\pi)=0$. A generic closed string vertex operator of the form $c \bar{c} V^{(h, h)}$, where $V^{(h, h)}$ is a bulk $(h, h)$ matter primary not depending on the $Y$ direction, can be formally put on-shell by going to a complexified mass shell. This can be done by multiplying $c \bar{c} V^{(h, h)}$ with $e^{2 i \sqrt{1-h} Y}$ whose conformal weight is $(1-h, 1-h)$. For $h>1$ (typical case) this is a negative weight primary which is in general not normalizable due to the divergent zero mode integration in the world-sheet path integral. But this is not a problem with Dirichlet boundary conditions, as the zero mode path integral will be localized at $y=0$. Moreover, since disk one point functions of bulk exponential operators are nonvanishing with Dirichlet boundary conditions, the corresponding modified tadpoles will also be generically nonzero.

\subsubsection{Lifting solutions}

The above example suggests that given a solution $\Psi$ we can consider adding an auxiliary $\mathrm{BCFT}^{\text {aux }}$ sector of total $c=0$ to the basis states of the original $\mathrm{BCFT}_{0}$ and search for a minimal extension or a lift of the solution, so that it becomes a solution in the lifted OSFT defined on

$$
\mathrm{BCFT}_{0}^{\prime} \equiv \mathrm{BCFT}_{0} \otimes \mathrm{BCFT}_{0}^{\mathrm{aux}}
$$

with a lifted BRST charge

$$
Q \rightarrow \tilde{Q} \equiv \sum_{n}: c_{-n}\left(L_{n}^{\text {matter }}+L_{n}^{\text {aux }}+\frac{1}{2} L_{n}^{\text {ghost }}\right): .
$$

If $\Psi$ describes new boundary conditions $\mathrm{BCFT}_{\Psi}$, we search for a solution $\tilde{\Psi}$ to the lifted equation

$$
\tilde{Q} \tilde{\Psi}+\tilde{\Psi} * \tilde{\Psi}=0
$$


such that it describes the boundary conditions $\mathrm{BCFT}_{\Psi} \otimes \mathrm{BCFT}_{0}^{\mathrm{aux}}$, i.e. it doesn't change the boundary conditions in the auxiliary BCFT. We expect that this requirement can be achieved by imposing that $\tilde{\Psi}$ depends on auxiliary degrees of freedom only through Virasoro operators. This expectation is supported by the many analytic and numerical examples we have studied, although we don't have an explicit proof of this.

Our ansatz for the lifted solution is thus

$$
\tilde{\Psi}=\sum_{M} \Psi_{M} \otimes L_{-M}^{\mathrm{aux}}|0\rangle^{\mathrm{aux}}
$$

where $M$ is a multi-index of the form

$$
M=\left\{m_{k}, \ldots, m_{1}\right\}, \quad m_{k} \geq m_{k-1} \geq \ldots \geq m_{1} \geq 2,
$$

and $L_{-M}$ stand for the corresponding product of negatively moded Virasoros

$$
L_{-M} \equiv L_{-m_{k}} \ldots L_{-m_{1}}
$$

The $\Psi_{M}$ 's are ghost number one states in the original $\mathrm{BCFT}_{0}$. The above expression defines the state $\tilde{\Psi}$ in terms of level expansion with respect to $L_{0}^{\text {aux }}$. The $c=0$ nature of the auxiliary CFT, together with the conservation laws for the star product and the form of the lifted BRST charge (2.2), implies that the equation of motion in the tensor theory reduces to the equation of motion in the original theory. Concretely, whenever we have a lifted solution $\tilde{\Psi}$, we can recover the original solution $\Psi$ by just looking at the auxiliary-level-zero part of $\tilde{\Psi}$, that is the part of $\tilde{\Psi}$ which is proportional to the auxiliary $\mathrm{SL}(2, \mathrm{R})$ vacuum. Denoting the $L_{0}^{\text {aux }}=0$ part of $\tilde{\Psi}$ as $\Psi$

$$
\tilde{\Psi}=\Psi \otimes|0\rangle^{\text {aux }}+\left(L_{0}^{\text {aux }} \geq 2 \text { terms }\right),
$$

one can easily show that

$$
\tilde{Q} \tilde{\Psi}+\tilde{\Psi} * \tilde{\Psi}=0
$$

implies

$$
Q \Psi+\Psi * \Psi=0 .
$$

This is so, since the product $L_{-M}^{\text {aux }}|0\rangle^{\text {aux }} * L_{-N}^{\text {aux }}|0\rangle^{\text {aux }}$ does not contain the vacuum $|0\rangle^{\text {aux }}$ unless both $M$ and $N$ are empty sets of indices.

It is tempting to think that to find such a lifted solution it should be enough to simply change all $L^{\text {matter }}$ into $L^{\text {matter }}+L^{\text {aux }}$ inside the level expansion (1.2) of the original solution $\Psi$. However, closer inspection reveals that the equation of motion of string field theory is satisfied, in general, due to cancellations between descendants of primaries arising from conformal transformation in the three-vertex and from the BRST charge on one hand, 
and descendants appearing in the operator product expansion of primary operators on the other hand 3

To appreciate the problem, focus on a marginal deformation generated by $\partial X$ to second order in the deformation parameter $\lambda$ in Siegel gauge

$$
\Psi_{\lambda}=\lambda c \partial X(0)|0\rangle-\lambda^{2} \frac{b_{0}}{L_{0}}(c \partial X|0\rangle * c \partial X|0\rangle)+O\left(\lambda^{3}\right) .
$$

To lift the solution to $\mathrm{BCFT}_{0}^{\prime}$ we demand for simplicity that $\partial X$ lifts to itself (we expect that more complicated lifts do not lead to factorized boundary state). Because of the Siegel gauge condition, the lifted solution is then uniquely specified by the first order in $\lambda$ term,

$$
\tilde{\Psi}_{\lambda}=\lambda c \partial X(0)|0\rangle-\lambda^{2} \frac{b_{0}}{L_{0}+L_{0}^{\text {aux }}}(c \partial X|0\rangle * c \partial X|0\rangle)+O\left(\lambda^{3}\right) .
$$

To evaluate the star product $c \partial X|0\rangle * c \partial X|0\rangle=\widehat{U}_{3} \widetilde{c \partial X}\left(\frac{\pi}{4}\right) \widetilde{c \partial X}\left(-\frac{\pi}{4}\right)|0\rangle$ (for the notation see [22]) one needs the OPE of $\partial X$ with itself. To lowest order in the level expansion one finds a coefficient times the identity operator and in the next-to-leading order the world-sheet energy momentum tensor $T^{X}$ in the free boson $\mathrm{BCFT}^{X}$ with $c=1$. In the total matter BCFT, $T^{X}$ decomposes as

$$
T^{X}=\frac{25}{26}\left(T^{X}-\frac{1}{25} T^{\prime}\right)+\frac{1}{26}\left(T^{X}+T^{\prime}\right),
$$

where $T^{\prime}$ denotes the energy momentum tensor of the rest of matter CFT with $c=25$. The first term is a conformal primary, the second is a descendant of the identity operator. So if we were lifting all the descendants via $T^{\text {matter }} \rightarrow T^{\text {matter }}+T^{\text {aux }}$, we would have to change also the primary $\left(T^{X}-\frac{1}{25} T^{\prime}\right) \rightarrow\left(T^{X}-\frac{1}{25} T^{\prime}\right)-\frac{1}{25} T^{\text {aux }}$, to keep the OPE of $\partial X$ with itself preserved. Had we just blindly applied $L^{\text {matter }} \rightarrow L^{\text {matter }}+L^{\text {aux }}$ to the level expansion (1.2) of the original solution, we would have violated the equation of motion in the lifted theory. Notice however that if we level expand the solution in the $\mathrm{BCFT}^{(X)} \otimes \mathrm{BCFT}^{\prime}$ basis, the geometrical lifting (2.11) is equivalent to systematically changing all $L_{N}^{\prime}$ 's with $\left(L^{\prime}+L^{\text {aux }}\right)_{N}$.

The above compatibility condition with the star product is not so easy to solve in the most generic situation but, at present, we have identified two (overlapping) families of string fields which can be straightforwardly lifted.

The first class is the algebra of wedge states with matter primary insertions, together with insertions of the $c$-ghost with its worldsheet derivatives, and line-integrals of the

\footnotetext{
${ }^{3}$ Analogously, the simple prescription $L^{\text {matter }} \rightarrow L^{\text {matter }}+L^{\text {aux }}$ would fail if the equations of motion were satisfied only up to nontrivial null-vectors in the Verma module of the identity.
} 
$b$-ghost 4 . This family is rich enough to contain all known analytic solutions. A generic element of the algebra takes the form

$$
\Phi=\sum_{i} F_{i} c B G_{i} c H_{i}
$$

where the ghost number zero string fields $F, G, H$ are star products of elements of the wedge algebra and matter primary insertions $\phi_{i}$,

$$
F_{i}=f_{1}(K) \phi_{1} \ldots f_{n_{i}}(K) \phi_{n_{i}}
$$

with the same generic expression for $G$ and $H$. In this case it is not difficult to realize that all multiplicative and differential properties are left intact by defining the lifted string field as

$$
\left.\tilde{\Phi} \equiv \Phi\right|_{K \rightarrow K+K^{\text {aux }}} .
$$

When level expanded, the above string field has the general structure given in (2.4). Notice however that due to the non trivial OPE between the matter insertions $\phi_{i}$, the level expansion of the lifted string field cannot be obtained by just applying the simple prescription $L^{\text {matter }} \rightarrow L^{\text {matter }}+L^{\text {aux }}$ to the level expansion of the string field before the lift (1.2). It is only when no matter primary insertion enters the game (as it is for the universal solutions) that the simple prescription $L^{\text {matter }} \rightarrow L^{\text {matter }}+L^{\text {aux }}$ in the level expansion of the solution is guaranteed to lift solutions to solutions.

The other family of simply liftable string fields is given whenever the matter CFT is the tensor product of two factors $\mathrm{CFT}_{1} \otimes \mathrm{CFT}_{2}$, with $c_{\mathrm{CFT}_{2}} \geq 1$ and with only $\mathrm{BCFT}_{1}$ primaries switched on 5 At ghost number one (relevant for string field theory solutions), this means that we consider a subspace of states of the form

$$
\Phi=\sum_{i} \sum_{N, M, P} \Phi_{N M P}^{i} L_{-N}^{(1)} L_{-M}^{(2)} L_{-P}^{\text {ghost }} c \phi_{i}^{(1)}(0)|0\rangle,
$$

where $\phi_{i}^{(1)}(x)$ are primary boundary fields of $\mathrm{BCFT}_{1}$. Now, if a $\Phi$ of this form is a solution (as it is the case, for example, for the numerical lump solutions, with the exception of the D-instanton), the corresponding lifted solution can be easily obtained by just replacing

$$
L^{(2)} \rightarrow L^{(2)}+L^{\mathrm{aux}}
$$

This is easily seen by noticing that, as far as the $\mathrm{BCFT}_{2}$ sector is concerned, the star product is completely determined by the conservation laws which only depend on the

\footnotetext{
${ }^{4}$ Obviously, by reparametrization, the wedge algebra can be replaced by any other surface state algebra.

${ }^{5}$ Aside of the induced non-diagonal primaries formed using operators from $\mathrm{CFT}_{1}$ and Virasoro descendants of the identity from $\mathrm{CFT}_{2}$. The condition $c_{\mathrm{CFT}_{2}} \geq 1$ is there to avoid the presence of non trivial null states on the Verma module on the identity.
} 
central charge, which is not changed by the lift. Again, had some primary been switched on in $\mathrm{BCFT}_{2}$, the simple lifting procedure we just advocated would fail because the OPE between the switched-on primaries would not be preserved by the lift, and the star product would not commute with the lift. As a final comment notice that, if $\mathrm{BCFT}_{2}$ contains a $c=1$ free boson with Neumann boundary conditions (as it is usually the case in known string field theory constructions), one can equivalently change the boundary conditions of this factor from Neumann to Dirichlet and still have a solution to the equation of motion, without explicitly tensoring an auxiliary BCFT. The relevant computations for the boundary state are insensitive to whether we uplift a spectator sector or whether we change boundary condition of a spectator direction to Dirichlet. We will use the latter simple shortcut in some explicit examples later in the paper.

To summarize, we search for a lifted solution of the form (2.4). We don't have a completely general analytic procedure to get the higher lifted components $\Psi_{M}$, if nothing is assumed on the starting solution $\Psi=\Psi_{\emptyset}$. However, in the case the solution $\Psi$ is made of surfaces with matter primary insertions, then the simplest lift is given by (2.15), and the corresponding coefficients $\Psi_{M}$ can be systematically computed by usual methods, if one needs to. Alternatively, when the solution lives in a BCFT which is the tensor product of two factors, and no primaries are switched on in one of the two factors (the spectator sector), as in (2.16), then the level expansion of the lifted solution is given by (2.17). Notice that the two lifts coincide for analytic solutions of the form (2.13) with a spectator sector.

A more general explicit constructive procedure for the $\Psi_{M}$ 's is needed, for example, for numerical marginal deformations along generic directions or more fundamentally for numerical D-instanton lumps, where there are no spectator dimensions nor a simple geometric picture for the solution. In such a case, however, one can construct the uplifted solution numerically. Since the original equations of motion are a subset of the lifted equations of motion, level by level, we can uniquely link the numerical solutions of the lifted theory to the corresponding solutions of the original theory by matching the coefficients in the $L_{0}^{\text {aux }}=0$ sector. This is an explicit construction of the lift for the numerical solution which, however, we have not yet tested against explicit examples.

\subsubsection{Lifting closed string states}

Given any CFT bulk primary of the form

$$
\mathcal{V}^{\alpha}(z, \bar{z})=c \bar{c} V^{\alpha}(z, \bar{z})
$$

where $V^{\alpha}$ is a purely matter primary of weight $\left(h_{\alpha}, h_{\alpha}\right)$, we can consider a formal bulk primary in $\mathrm{CFT}^{\text {aux }}, w^{\alpha}(z, \bar{z})$ of weight $\left(1-h_{\alpha}, 1-h_{\alpha}\right)$ with the property that

$$
\left\langle w^{\alpha}(0)\right\rangle_{\text {disk }}^{\mathrm{BCFT}^{\mathrm{aux}}}=1, \quad \forall \alpha .
$$


Explicitly, as discussed in more detail in appendix $\mathrm{A}$, we can define BCFT ${ }^{\text {aux }}$ to be the tensor product of a free boson $Y$ with Dirichlet boundary conditions $(c=1)$ and a linear dilaton $\varphi$ with background charge $Q=\frac{1}{\sqrt{3}}$ with Neumann boundary conditions and $c=1-6 Q^{2}=-1$. In this case we can systematically take

$$
w^{\alpha}=e^{2 i \sqrt{1-h_{\alpha}} Y} e^{\frac{2 i}{\sqrt{3}} \varphi},
$$

which has weight $\left(1-h_{\alpha}, 1-h_{\alpha}\right)$ and satisfies (2.19), thanks to the Dirichlet conditions for $Y$ and the saturation of the background charge on the disk. Notice that, for $h_{\alpha}>1$, $w^{\alpha}$ is not normalizable in the auxiliary closed string Hilbert space, but still it has a well defined one-point function on the disk. Other choices of BCFT ${ }^{\text {aux }}$ are clearly possible.

\subsubsection{Generalized Ellwood invariant}

For OSFT purposes the closed string insertion

$$
\tilde{\mathcal{V}}^{\alpha} \equiv c \bar{c} V^{\alpha} \otimes w^{\alpha}
$$

will be a total $(0,0)$ bulk primary (in fact, a formal, not normalizable, element of the $\tilde{Q}$ closed string cohomology). Thus, assuming Ellwood conjecture, the Ellwood invariant will compute the difference in the tadpoles between the two BCFT's related by the classical solution. But since the solution $\tilde{\Psi}$ does not switch on any new primaries in $\mathrm{BCFT}_{0}^{\prime}$, the generalized Ellwood invariant will be proportional to the disk one-point function of $w^{\alpha}$

$$
\begin{aligned}
& -4 \pi i\left\langle E\left[\tilde{\mathcal{V}}^{\alpha}\right] \mid \tilde{\Psi}\right\rangle^{\mathrm{BCFT}_{0}^{\prime}} \\
& =\left\langle\tilde{\mathcal{V}}^{\alpha}\left|c_{0}^{-}\right| \tilde{B}_{\tilde{\Psi}}\right\rangle-\left\langle\tilde{\mathcal{V}}^{\alpha}\left|c_{0}^{-}\right| \tilde{B}_{0}\right\rangle \\
& =\left(\left\langlec \bar{c} V^{\alpha}\left|\otimes\left\langle w^{\alpha}\right|\right) c_{0}^{-}\left(\left|B_{\Psi}\right\rangle \otimes\left|B_{\text {aux }}\right\rangle\right)-\left(\left\langlec \bar{c} V^{\alpha}\left|\otimes\left\langle w^{\alpha}\right|\right) c_{0}^{-}\left(\left|B_{0}\right\rangle \otimes\left|B_{\text {aux }}\right\rangle\right)\right.\right.\right.\right. \\
& =\left(\left\langle c \bar{c} V^{\alpha}\left|c_{0}^{-}\right| B_{\Psi}\right\rangle-\left\langle c \bar{c} V^{\alpha}\left|c_{0}^{-}\right| B_{0}\right\rangle\right) \times\left\langle w^{\alpha}(0)\right\rangle_{\text {disk }}^{\text {BCFT }} \text { aux } \\
& =\left\langle c \bar{c} V^{\alpha}\left|c_{0}^{-}\right| B_{\Psi}\right\rangle-\left\langle c \bar{c} V^{\alpha}\left|c_{0}^{-}\right| B_{0}\right\rangle .
\end{aligned}
$$

Notice that the auxiliary CFT disappeared from the RHS. Conveniently, we can relate the $\mathrm{BCFT}_{0}$-boundary state with the Ellwood invariant of the lifted tachyon vacuum, $\tilde{\Psi}_{T V}$, and we can write the 'generalized' Ellwood conjecture in the simple form

$$
\left\langle c \bar{c} V^{\alpha}\left|c_{0}^{-}\right| B_{\Psi}\right\rangle=-4 \pi i\left\langle E\left[\tilde{\mathcal{V}}^{\alpha}\right] \mid \tilde{\Psi}-\tilde{\Psi}_{T V}\right\rangle
$$

String field theory solutions related by gauge transformations should describe the same BCFT and thus the same boundary state $\left|B_{\Psi}\right\rangle$. Although the right hand side is 
manifestly invariant under the gauge transformations in the new OSFT based on $\mathrm{BCFT}_{0}^{\prime}$, to show that it is invariant also under the gauge transformation in the original OSFT based on $\mathrm{BCFT}_{0}$ requires a little thought. One has to show that the lifting from $\mathrm{BCFT}_{0}$ to $\mathrm{BCFT}_{0}^{\prime}$ commutes with gauge transformations. This is easily arguable in the following way. Suppose we have a solution $\Psi$ and its lift $\tilde{\Psi}$ written as (2.4). It is not difficult to realize that if we change $\Psi$ by a gauge transformation with group-element $U$

$$
\Psi^{\prime}=U^{-1}(Q+\Psi) U
$$

we can very easily get an infinite family of lifted solutions of the form (2.4) which are gauge equivalent to $\tilde{\Psi}$

$$
\tilde{\Psi}^{\prime}=\tilde{U}^{-1}(\tilde{Q}+\tilde{\Psi}) \tilde{U}
$$

It is enough to choose 6

$$
\tilde{U}=U \otimes|0\rangle^{\mathrm{aux}}+\sum_{M \neq \emptyset} U_{M} \otimes L_{-M}^{\mathrm{aux}}|0\rangle^{\mathrm{aux}},
$$

where the higher auxiliary components $U_{M}$ are generic ghost number zero string fields in $\mathrm{BCFT}_{0}$ (with the only obvious requirement that they must be chosen in such a way that $\tilde{U}$ is invertible). The $c=0$ nature of BCFT ${ }^{\text {aux }}$ assures that both the lifted BRST charge $\tilde{Q}$ and the star product behave in such a way that the auxiliary-level-zero element of $\tilde{\Psi}^{\prime}$ is nothing but $\Psi^{\prime}$

$$
\tilde{U}^{-1}(\tilde{Q}+\tilde{\Psi}) \tilde{U}=U^{-1}(Q+\Psi) U \otimes|0\rangle^{\text {aux }}+\left(L_{0}^{\text {aux }} \geq 2 \text { terms }\right) .
$$

Therefore gauge equivalent classes of solutions lift to gauge equivalent classes of lifted solutions.

\subsection{Ellwood invariants and Ishibashi states}

Using the Ellwood conjecture, we can compute the overlap

$$
\left\langle B_{\Psi}\left|c_{0}^{-}\right| \mathcal{V}\right\rangle
$$

for any liftable OSFT solution $\Psi$ and closed string state $|\mathcal{V}\rangle$ of the form

$$
|\mathcal{V}\rangle=V^{(h, h)}(0) c_{1} \bar{c}_{1}|0\rangle_{S L(2, C)}
$$

\footnotetext{
${ }^{6}$ This also shows that the lift is not unique: for any given solution $\Psi$ and its lift $\tilde{\Psi}$ one can always change the higher level components in the auxiliary sector of $\tilde{\Psi}$ with a gauge transformation $\tilde{U}$ whose $L_{0}^{\text {aux }}=0$ component does not change $\Psi$. The question remains if there are multiple liftings of the form (2.4) of the same solution which are not gauge equivalent and which might then give rise to different observables. On physical grounds we expect that this cannot happen.
} 
where $V^{(h, h)}(z, \bar{z})$ is a weight $(h, h)$ bulk primary in the matter sector. Such a state necessarily obeys

$$
\begin{aligned}
L_{n \geq 1}^{\text {matter }}|\mathcal{V}\rangle & =\bar{L}_{n \geq 1}^{\text {matter }}|\mathcal{V}\rangle=0 \\
c_{n \geq 1}|\mathcal{V}\rangle & =\bar{c}_{n \geq 1}|\mathcal{V}\rangle=0 \\
b_{n \geq 0}|\mathcal{V}\rangle & =\bar{b}_{n \geq 0}|\mathcal{V}\rangle=0
\end{aligned}
$$

Clearly, the closed string states $c \bar{c} V^{(h, h)}$ do not span the whole set of off-shell closed string fields. However, any generic off-shell closed string state can be obtained by acting with ghost oscillators and matter Virasoro generators on the ground states given by the $|\mathcal{V}\rangle$ 's. This choice of basis is quite convenient from the OSFT point of view, since the states $|\mathcal{V}\rangle$ directly enter in the Ellwood invariant. But it is also very convenient from the closed string point of view: the knowledge of the overlap $\left\langle\mathcal{V}\left|c_{0}^{-}\right| B_{\Psi}\right\rangle$ is just enough to define all overlaps with the boundary state.

This is because the boundary state is a ghost number three closed string state which describes conformal boundary conditions in the total matter and ghost Hilbert space. This is summarized by

$$
\begin{aligned}
b_{0}^{-}\left|B_{\Psi}\right\rangle & =0 \\
\left(L_{n}^{\text {tot }}-\bar{L}_{-n}^{\text {tot }}\right)\left|B_{\Psi}\right\rangle & =0 \\
\left(Q_{g h}-3\right)\left|B_{\Psi}\right\rangle & =0
\end{aligned}
$$

where $Q_{g h}$ is the total ghost number operator obeying $Q_{g h}|0\rangle_{S L(2, C)}=0$. We show in appendix C that these three (sets of) conditions by themselves already imply the standard gluing conditions

$$
\begin{aligned}
\left(b_{n}-\bar{b}_{-n}\right)\left|B_{\Psi}\right\rangle & =0, \\
\left(c_{n}+\bar{c}_{-n}\right)\left|B_{\Psi}\right\rangle & =0 \\
\left(L_{n}^{\text {matter }}-\bar{L}_{-n}^{\text {matter }}\right)\left|B_{\Psi}\right\rangle & =0, \\
(Q+\bar{Q})\left|B_{\Psi}\right\rangle & =0 .
\end{aligned}
$$

These gluing conditions allow to trade raising operators acting on the closed string state $|\mathcal{V}\rangle$ for lowering operators which will vanish upon acting on $|\mathcal{V}\rangle$. Thus any overlap of a closed string state $|W\rangle$ built by acting with raising operators on $|\mathcal{V}\rangle$ will be proportional to the corresponding overlap of the boundary state with $|\mathcal{V}\rangle$ itself, the constant of proportionality being a number which can easily be computed using the matter Virasoro algebra and the b,c oscillator algebra. Thus, up to automatic operations, the boundary state for a solution $\Psi$ is completely encoded in the (generalized) Ellwood invariants. 
We can beautifully and very efficiently formulate these observations in terms of the socalled Ishibashi states. Let $\left\{V_{\alpha}\right\}$ be the collection of non-singular spinless bulk primaries of weight $\left(h_{\alpha}, h_{\alpha}\right)$ in the matter $\mathrm{CFT} 7$

$$
\begin{aligned}
\left(L_{0}-\bar{L}_{0}\right)\left|V_{\alpha}\right\rangle & =\left(h_{\alpha}-h_{\alpha}\right)\left|V_{\alpha}\right\rangle=0 \\
L_{n}\left|V_{\alpha}\right\rangle & =\bar{L}_{n}\left|V_{\alpha}\right\rangle=0, \quad n \geq 1 .
\end{aligned}
$$

Let's define a BPZ-dual basis of primaries $\left\{V^{\beta}\right\}$ such that

$$
\left\langle V^{\alpha} \mid V_{\beta}\right\rangle=\delta_{\beta}^{\alpha}
$$

This is possible once singular (null) states have been projected out. To any spinless vertex operator $V_{\alpha}$ we can associate the corresponding conformal Ishibashi state, which (up to normalization) is the unique state $\left.\left|V_{\alpha}\right\rangle\right\rangle$ in the Virasoro Verma module of $V_{\alpha}$ satisfying the Virasoro gluing conditions

$$
\left.\left(L_{n}-\bar{L}_{-n}\right)\left|V_{\alpha}\right\rangle\right\rangle=0 .
$$

The explicit form of the Ishibashi state $\left.\left|V_{\alpha}\right\rangle\right\rangle$ to any desired level can be found easily by solving the gluing conditions in the Verma module of $V_{\alpha}$ level by level, and one finds in the absence of null states

$$
\begin{aligned}
\left.\left|V_{\alpha}\right\rangle\right\rangle=\left[\begin{array}{l}
1 \\
\quad+\frac{1}{2 h_{\alpha}} L_{-1} \bar{L}_{-1}
\end{array}\right. \\
\quad+B\left(h_{\alpha}, c\right)\left(2\left(1+2 h_{\alpha}\right) L_{-2} \bar{L}_{-2}-3\left(L_{-2} \bar{L}_{-1}^{2}+L_{-1}^{2} \bar{L}_{-2}\right)+\frac{8 h_{\alpha}+c}{4 h_{\alpha}} L_{-1}^{2} \bar{L}_{-1}^{2}\right) \\
\quad+\cdots]\left|V_{\alpha}\right\rangle, \\
B\left(h_{\alpha}, c\right)=\frac{1}{2 h_{\alpha}\left(8 h_{\alpha}-5\right)+c\left(2 h_{\alpha}+1\right)} .
\end{aligned}
$$

Had there been a null state at some level, the coefficients in this expression at that level would be divergent. For example the level 2 null state appears exactly for those values of $h$ and $c$ for which $B(h, c)$ diverges. In such a case one should exclude the null states from the Verma module. Solving then the gluing conditions with null states projected out gives analogous expression to (2.42) but with finite coefficients. A simple closed form of the solution to the gluing condition for the general case has been found by Ishibashi [8]

$$
\left.\left|V_{\alpha}\right\rangle\right\rangle=\sum_{n}|n, \alpha\rangle \otimes \overline{|n, \alpha\rangle}
$$

\footnotetext{
${ }^{7}$ In CFTs on noncompact target spaces $\alpha$ will in general be a continuous variable, like the momentum.
} 
where the sum runs over orthonormal basis of states in the irreducible representation of the chiral Virasoro algebra built over the primary $V_{\alpha}$. In writing this, we have assumed that the closed string primary $V_{\alpha}$ can be decomposed into the product of holomorphic and antiholomorphic parts. Relaxing this assumption [12], we can rewrite it equivalently as

$$
\left.\left|V_{\alpha}\right\rangle\right\rangle=\sum_{I J} M^{I J}\left(h_{\alpha}\right) L_{-I} \bar{L}_{-J}\left|V_{\alpha}\right\rangle
$$

where the indices $I, J$ label the non-degenerate descendants in the conformal family of $V_{\alpha}$, and $M^{I J}\left(h_{\alpha}\right)$ is defined as the inverse of the real symmetric matrix

$$
M_{I J}\left(h_{\alpha}\right)=\left\langle V^{\alpha}\left|L_{I} L_{-J}\right| V_{\alpha}\right\rangle=\left\langle V^{\alpha}\left|\bar{L}_{I} \bar{L}_{-J}\right| V_{\alpha}\right\rangle
$$

The normalization has been chosen so that

$$
\left.\left\langle V^{\alpha} \mid V_{\beta}\right\rangle\right\rangle=\left\langle V^{\alpha} \mid V_{\beta}\right\rangle=\delta_{\beta}^{\alpha}
$$

Any boundary state $\left|B_{*}\right\rangle$ in the matter CFT can therefore be written as

$$
\left.\left|B_{*}\right\rangle=\sum_{\alpha} n_{*}^{\alpha}\left|V_{\alpha}\right\rangle\right\rangle
$$

If we want to define $\mathrm{BCFT}_{*}$ through its boundary state, the coefficients $n_{*}^{\alpha}$ must be precisely chosen in order to satisfy Cardy conditions (open string analog of modular invariance) and sewing conditions (factorization of bulk $n$-point functions in open and closed string channels), see e.g. [13]. If the boundary state $\left|B_{*}\right\rangle$ is known, we can easily get $n_{*}^{\alpha}$ from

$$
n_{*}^{\alpha}=\left\langle V^{\alpha} \mid B_{*}\right\rangle .
$$

In the OSFT approach to BCFT, instead of searching for linear combinations of Ishibashi states obeying nontrivial consistency conditions, we search for solutions to the equation of motion. If OSFT is a consistent theory we expect such classical solutions to automatically describe consistent boundary conditions.

With the above premises, our proposal can be compactly written as

$$
\begin{gathered}
\left.\left|B_{\Psi}\right\rangle=\sum_{\alpha} n_{\Psi}^{\alpha}\left|V_{\alpha}\right\rangle\right\rangle \otimes\left|B_{g h}\right\rangle, \\
n_{\Psi}^{\alpha} \equiv 2 \pi i\left\langle E\left[\tilde{\mathcal{V}}^{\alpha}\right] \mid \tilde{\Psi}-\tilde{\Psi}_{T V}\right\rangle,
\end{gathered}
$$


where we used, see also appendix C,

$$
\begin{aligned}
\left\langle 0\left|c_{-1} \bar{c}_{-1} c_{0}^{-}\right| B_{g h}\right\rangle & =\left\langle\left(c_{0}-\bar{c}_{0}\right) c \bar{c}(0)\right\rangle_{\text {disk }}^{\text {ghost }}=-2, \\
\mathcal{V}^{\alpha} & =c \bar{c} V^{\alpha} \\
\tilde{\mathcal{V}}^{\alpha} & =\mathcal{V}^{\alpha} \otimes w^{\alpha}
\end{aligned}
$$

and $\Psi_{T V}$ is any OSFT solution for the tachyon vacuum whose contribution replaces the corresponding contribution from the $\mathrm{BCFT}_{0}$ boundary state.

\subsection{Ellwood invariants and boundary primaries}

It is useful and interesting to elucidate the relation between the primary boundary fields that are 'switched on' in an OSFT classical solution and the boundary state which is associated to the solution via our construction through the Ellwood conjecture. To this end we consider the solution expressed as

$$
\Psi-\Psi_{T V}=\sum_{j} \sum_{I, J} a_{I J}^{j} L_{-I}^{\text {matter }}\left|\phi_{j}\right\rangle \otimes L_{-J}^{\text {ghost }} c_{1}|0\rangle
$$

where $I, J$ are multi-indices of the form

$$
N=\left\{n_{k}, \ldots, n_{1}\right\}, \quad n_{k} \geq n_{k-1} \geq \ldots \geq n_{1} \geq 1
$$

and $L_{-I}^{\text {matter }}, L_{-J}^{\text {ghost }}$ stand for the corresponding products of negatively moded Virasoros

$$
L_{-N} \equiv L_{-n_{k}} \ldots L_{-n_{1}}
$$

acting respectively on the matter primary $\left|\phi_{j}\right\rangle$ and the unique ghost primary $c_{1}|0\rangle$ at ghost number one. The lifted solution in $\mathrm{BCFT}_{0}^{\prime}=\mathrm{BCFT}_{0} \otimes \mathrm{BCFT}^{\text {aux }}$ will be given by

$$
\tilde{\Psi}-\tilde{\Psi}_{T V}=\sum_{j} \sum_{I, J, M} a_{I J M}^{j} L_{-I}^{\text {matter }}\left|\phi_{j}\right\rangle \otimes L_{-J}^{\text {ghost }} c_{1}|0\rangle \otimes L_{-M}^{\text {aux }}|0\rangle^{\text {aux }},
$$

where the lifted coefficients $a_{I J M}$ reduce to the original ones $a_{I J}$ when the multi-index $M$ is the empty set.

In computing generalized Ellwood invariant $\left\langle E\left[\tilde{\mathcal{V}}^{\beta}\right] \mid \tilde{\Psi}-\tilde{\Psi}_{T V}\right\rangle$ associated to the closed string field

$$
\tilde{\mathcal{V}}^{\beta}=\mathcal{V}^{\beta} \otimes w^{\beta}=c \bar{c} V^{\beta} \otimes w^{\beta},
$$

where $w^{\beta}$ is a weight $\left(1-h_{\beta}\right)$ primary in the $c=0$ BCFT ${ }^{\text {aux }}$ with one-point function on the disk normalized to unity (see appendix $(\mathrm{A})$, it is useful to consider the conservation laws of the anomalous derivations

$$
K_{n}=L_{n}-(-1)^{n} L_{-n},
$$


in matter, ghost and auxiliary sectors separately. These conservation laws can be found in [7] and an alternative simple derivation is offered in appendix B. For instance, in the matter sector, the law takes the form

$$
\begin{aligned}
& \left\langle E\left[\tilde{\mathcal{V}}^{\beta}\right]\right| K_{2 n+1}^{\text {matter }}=0 \\
& \left\langle E\left[\tilde{\mathcal{V}}^{\beta}\right]\right| K_{2 n}^{\text {matter }}=n(-1)^{n}\left(13-16 h_{\beta}\right)\left\langle E\left[\tilde{\mathcal{V}}^{\beta}\right]\right| .
\end{aligned}
$$

Thanks to these conservation laws one can get rid of all the Virasoros in the solution, level by level. It follows that 8

$$
\left\langle E\left[\tilde{\mathcal{V}}^{\beta}\right] \mid \tilde{\Psi}-\tilde{\Psi}_{T V}\right\rangle=\sum_{j} A_{\Psi}^{\beta j}\left\langle E\left[\tilde{\mathcal{V}}^{\beta}\right] \mid c \phi_{j}\right\rangle^{\mathrm{BCFT} T_{0}^{\prime}}
$$

where $A_{\Psi}^{\beta j}$ is a gauge invariant linear combination 9 of the coefficients $a_{I J M}^{j}$ appearing in the lifted solution (2.58). Using the conservation laws of appendix B recursively we can write them explicitly as

$$
A_{\Psi}^{\beta j}=\sum_{I, J, M} K_{I J M}^{\left(h_{\beta}, h_{j}\right)} a_{I J M}^{j}
$$

Notice that the constants $K^{\left(h_{j}, h_{\beta}\right)}$ depend only on the weights of the matter closed string state $V^{\beta}$ and of the boundary primary state $\phi_{j}$, and otherwise are completely universal. Obviously, the coefficients $A_{\Psi}^{\beta j}$ do not depend on whether the solution $\Psi$ is expressed in the basis of Virasoro generators, or matter and ghost oscillators. In the latter case, analogous formulas can be obtained by applying the oscillator conservation laws, which we derive in appendix $\mathrm{B}$ too. The final result is exactly the same, level by level, as the one obtained using Virasoro generators.

\footnotetext{
${ }^{8}$ Since we are computing an Ellwood invariant, the tachyon vacuum solution can be traded for the simple string field

$$
\tilde{\Psi}_{T V} \rightarrow \frac{2}{\pi} c_{1}|0\rangle
$$

Thus, in the Ellwood invariant, the difference between $\Psi$ and $\left(\Psi-\Psi_{T V}\right)$ only appears as a universal shift in the coefficient of the zero momentum tachyon. Notice in particular that for the perturbative vacuum we get $A_{P V}^{\beta 1}=-\frac{2}{\pi}$ as the only nonvanishing coefficient.

${ }^{9}$ The gauge invariance of the coefficients follows from the gauge invariance of the left hand side, assuming that no two boundary operators have the same bulk-boundary two-point function for every bulk operator.
} 
Computing the Ellwood invariant we find

$$
\begin{aligned}
-4 \pi i\left\langle E\left[\tilde{\mathcal{V}}^{\beta}\right] \mid c \phi_{j}\right\rangle^{\mathrm{BCFT}_{0}^{\prime}} & =-4 \pi i\left(\frac{2}{\pi}\right)^{h_{j}-1}\left\langle\tilde{\mathcal{V}}^{\beta}(i \infty) c \phi_{j}(0)\right\rangle_{C_{1}}^{\mathrm{BCFT}_{0}^{\prime}} \\
& =-4 \pi i\left(\frac{2}{\pi}\right)^{h_{j}-1} \frac{1}{2 \pi i}|2 \pi i|^{h_{j}}\left\langle\tilde{\mathcal{V}}^{\beta}(0) c \phi_{j}(1)\right\rangle_{\mathrm{disk}}^{\mathrm{BCFT}_{0}^{\prime}} \\
& =-\pi 4^{h_{j}}\left\langle\tilde{\mathcal{V}}^{\beta}(0) c \phi_{j}(1)\right\rangle_{\mathrm{disk}}^{\mathrm{BCFT}_{0}^{\prime}} \\
& =\pi 4^{h_{j}}\left\langle V^{\beta}(0) \phi_{j}(1)\right\rangle_{\text {disk }}^{\mathrm{BCFT}_{0}^{\text {matter }}}
\end{aligned}
$$

where we used (2.19) and

$$
\langle c \bar{c}(0) c(1)\rangle_{\text {disk }}^{\text {ghost }}=-1 .
$$

Notice that $\left\langle V^{\beta}(0) \phi_{j}(1)\right\rangle$ is the basic bulk-boundary two-point function of the matter part of $\mathrm{BCFT}_{0}$. Using this we can express the coefficients in front of the Ishibashi states $\left.\left|V_{\beta}\right\rangle\right\rangle$ in the $\left|B_{\Psi}\right\rangle$ boundary state in terms of the bare bones

$$
n_{\Psi}^{\beta}=-\frac{\pi}{2} \sum_{j} 4^{h_{j}} A_{\Psi}^{\beta j}\left\langle V^{\beta}(0) \phi_{j}(1)\right\rangle_{\mathrm{disk}}^{\mathrm{BCFT}_{0}^{\text {matter }}} .
$$

As a consistency check we derive the same formula directly on the upper half plane

$$
\begin{aligned}
-4 \pi i\left\langle E\left[\tilde{\mathcal{V}}^{\beta}\right] \mid c \phi_{j}\right\rangle^{\mathrm{BCFT}_{0}^{\prime}} & =-4 \pi i\left\langle\tilde{V}^{\beta}(i,-i) f_{I} \circ c \phi_{j}(0)\right\rangle_{\mathrm{UHP}}^{\mathrm{BCFT}_{0}^{\prime}} \\
& =-4 \pi i\left(f_{I}^{\prime}(0)\right)^{h_{j}-1}\left\langle\tilde{V}^{\beta}(i,-i) c \phi_{j}(0)\right\rangle_{\mathrm{UHP}}^{\mathrm{BCFT}_{0}^{\prime}} \\
f_{I}(z) & =\frac{2 z}{1-z^{2}}
\end{aligned}
$$

Factorizing the correlator in ghost, matter and the auxiliary sectors

$$
\left\langle\tilde{V}^{\beta}(i,-i) c \phi_{j}(0)\right\rangle_{\mathrm{UHP}}^{\mathrm{BCFT}_{0}^{\prime}}=\langle c(i) c(-i) c(0)\rangle_{\mathrm{UHP}}\left\langle V^{\beta}(i,-i) \phi_{j}(0)\right\rangle_{\mathrm{UHP}}\left\langle w^{\beta}(i,-i)\right\rangle_{\mathrm{UHP}},
$$

we find

$$
\begin{aligned}
\langle c(i) c(-i) c(0)\rangle_{\mathrm{UHP}} & =2 i \\
\left\langle w^{\beta}(i,-i)\right\rangle_{\mathrm{UHP}} & =4^{h_{\beta}-1}\left\langle w^{\beta}(0,0)\right\rangle_{\mathrm{disk}}=4^{h_{\beta}-1}
\end{aligned}
$$

where use of (2.19) has been made. In total we thus get

$$
n_{\Psi}^{\beta}=-\frac{\pi}{2} \sum_{j} 2^{h_{j}} 4^{h_{\beta}} A_{\Psi}^{\beta j}\left\langle V^{\beta}(i,-i) \phi_{j}(0)\right\rangle_{\mathrm{UHP}}^{\mathrm{BCFT}_{0}^{\text {matter }}}
$$


Consistently we find

$$
\begin{aligned}
\left\langle V^{\beta}(i,-i) \phi_{j}(0)\right\rangle_{\mathrm{UHP}} & =2^{h_{j}} 4^{-h_{\beta}}\left\langle V^{\beta}(0) \phi_{j}(1)\right\rangle_{\text {disk }} \\
& =\left|f^{\prime}(0)\right|^{h_{j}}\left|f^{\prime}(i)\right|^{2 h_{\beta}}\left\langle V^{\beta}(0) \phi_{j}(1)\right\rangle_{\text {disk }} \\
f(z) & =\frac{1+i z}{1-i z} .
\end{aligned}
$$

Notice that the formulas (2.66) or (2.72) explicitly express the boundary state in terms of the $\mathrm{BCFT}_{0}$ primaries that are switched on in the solution $\Psi$. As we will see later on, this is very useful for identification of the boundary conditions described by any liftable numerical solution which, level by level, can always be put to the form (2.55).

\section{Analytic solutions: Rolling tachyon}

The aim of this section is to illustrate our construction in an explicit case where Ellwood conjecture has been verified, and all OSFT computations have been done already. We select the simplest well-defined OSFT solutions corresponding to marginal deformations of the initial $\mathrm{BCFT}_{0}$, where the marginal current has regular OPE with itself. The whole construction can be readily extended [5] to the Kiermaier-Okawa solutions [14, 15], as well as to any other example in which the Ellwood invariant has been shown to analytically compute the tadpole shift, for example [16, 17]. For definiteness we select the rolling tachyon marginal deformation generated by the marginal current $V=e^{X^{0}}$.

These solutions have been constructed in the $\mathcal{B}_{0}$-gauge in [18, 19] and extended to more general gauges in [20, 21]

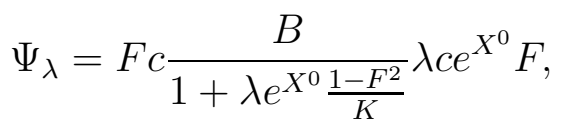

where $F=F(K)^{10}$ and $K, B, c$ are the familiar string fields [22, 23, 24, 25, 26], and $e^{X^{0}}$ is the insertion of the exactly marginal boundary operator $: e^{X^{0}}:(s)$ in the sliver frame.

Given an on-shell weight-zero primary closed string state $\mathcal{V}=c \bar{c} V^{(1,1)}$, the Ellwood invariant for this class of solutions has been computed in three different ways [27, 28, 29], and the result (with the $\mathrm{BCFT}_{0}$ contribution - given by the tachyon vacuum invariantconveniently subtracted) is

$$
\begin{aligned}
\left\langle E[\mathcal{V}] \mid \Psi-\Psi_{T V}\right\rangle & =-\left\langle e^{-\lambda \int_{0}^{1} d s e^{X^{0}}(s)} \mathcal{V}(i \infty) c(0)\right\rangle_{C_{1}}^{\mathrm{BCFT}_{0}} \\
& =-\frac{1}{2 \pi i}\left\langle e^{-\lambda \int_{0}^{2 \pi} d \theta e^{X^{0}}\left(e^{i \theta}\right)} \mathcal{V}(0) c(1)\right\rangle_{\text {disk }}^{\mathrm{BCFT}_{0}}
\end{aligned}
$$

\footnotetext{
${ }^{10}$ We assume the conditions $F(0)=1, F^{\prime}(0)<0$ and $F(\infty)=0$.
} 
The nontrivial rearrangement of the $e^{X^{0}}$ insertions in the solution into a simple boundary interaction is a general consequence of the particular form of the solution and the string field $F(K)$, as discussed in [29, 30].

This closed string tadpole is in fact closely related to the proper overlap of a closed string of the form $c \bar{c} V_{m}$ with the boundary state of $\Psi$

$$
\begin{aligned}
\left\langle e^{-\lambda \int_{0}^{2 \pi} d \theta e^{X^{0}}\left(e^{i \theta}\right)} c \bar{c} V_{m}^{(h, h)}(0) c(1)\right\rangle_{\text {disk }}^{\mathrm{BCFT}_{0}} & =\frac{1}{2}\left\langle e^{-\lambda \int_{0}^{2 \pi} d \theta e^{X^{0}}\left(e^{i \theta}\right)}\left(c_{0}-\bar{c}_{0}\right) c \bar{c} V_{m}^{(h, h)}(0)\right\rangle_{\mathrm{disk}}^{\mathrm{BCFT}_{0}} \\
& \equiv \frac{1}{2}\left\langle B_{\Psi}\left|c_{0}^{-}\right| c \bar{c} V_{m}^{(h, h)}\right\rangle
\end{aligned}
$$

where in the last line we have used the defining expression for the boundary state, in particular in the ghost sector we used (C.42). Notice, that although this relation is trivially true for any matter operator, there is no gauge invariant observable in the OSFT defined on $\mathrm{BCFT}_{0}$ (with generic boundary conditions) that could give the LHS of (3.3) for $h \neq 1$. To overcome this difficulty we lift the solution to the OSFT based on

$$
\mathrm{BCFT}_{0}^{\prime}=\mathrm{BCFT}_{0} \otimes \mathrm{BCFT}^{\mathrm{aux}}
$$

Because of the geometric nature of the solution (3.1), the simplest lifting we can do is to replace the $\mathrm{BCFT}_{0}$ worldsheet generated by $K$ with the $\mathrm{BCFT}_{0}^{\prime}$ one, generated by $K+K^{\text {aux }}$,i.e 11

$$
\tilde{\Psi}=\left.\Psi\right|_{K \rightarrow K+K^{\text {aux }}} .
$$

Consider now $\mathcal{V}^{(h)}=c \bar{c} V^{(h, h)}$, where $V^{(h, h)}$ is a weight $h$ level-matched primary of BCFT . $_{0}$ The state can be turned into a weight zero primary

$$
\tilde{\mathcal{V}}^{(h)}=\mathcal{V}^{(h)} e^{2 \sqrt{h-1} Y} e^{\frac{2 i \phi}{\sqrt{3}}}
$$

which now has a nonvanishing tadpole in $\mathrm{BCFT}_{0}^{\prime}$, chosen as in section 2.1.

Now we compute an Ellwood invariant in this slightly modified OSFT

$$
\begin{aligned}
& \left\langle I\left|\tilde{\mathcal{V}}^{(h)}(i)\right| \tilde{\Psi}-\tilde{\Psi}_{T V}\right\rangle=-\left\langle e^{-\lambda \int_{0}^{1} d s e^{X^{0}}(s)} \tilde{\mathcal{V}}^{(h)}(i \infty) c(0)\right\rangle_{C_{1}}^{\mathrm{BCFT}_{0}^{\prime}} \\
& =-\frac{1}{2 \pi i}\left\langle e^{-\lambda \int_{0}^{2 \pi} d \theta e^{X^{0}}\left(e^{i \theta}\right)} \tilde{\mathcal{V}}^{(h)}(0) c(1)\right\rangle_{\text {disk }}^{\mathrm{BCFT}_{0}^{\prime}} \\
& =-\frac{1}{2 \pi i}\langle c \bar{c}(0) c(1)\rangle\left\langle e^{-\lambda \int_{0}^{2 \pi} d \theta e^{X^{0}}\left(e^{i \theta}\right)} V^{h, h}(0)\right\rangle^{\mathrm{BCFT}_{0}}\left\langle e^{2 \sqrt{h-1} Y} e^{\frac{2 i \phi}{\sqrt{3}}}(0)\right\rangle^{\mathrm{BCFT}^{\text {aux }}} \\
& =-\frac{1}{4 \pi i}\left\langle e^{-\lambda \int_{0}^{2 \pi} d \theta e^{X^{0}}\left(e^{i \theta}\right)}\left(c_{0}-\bar{c}_{0}\right) c \bar{c} V^{h, h}(0)\right\rangle_{\mathrm{disk}}^{\mathrm{BCFT}_{0}},
\end{aligned}
$$

\footnotetext{
${ }^{11}$ We could have equivalently selected a space-like direction $Y$ (along which no boundary primaries are excited) and change its boundary conditions to Dirichlet.
} 
where we have used (2.19). We thus found

$$
\begin{aligned}
\left\langle c \bar{c} V^{(h, h)}\left|c_{0}^{-}\right| B_{\Psi}\right\rangle & =-4 \pi i\left\langle I\left|\tilde{\mathcal{V}}^{(h)}(i)\right| \tilde{\Psi}-\tilde{\Psi}_{T V}\right\rangle \\
& =\left\langle c \bar{c} V^{(h, h)}(0)\left(c_{0}-\bar{c}_{0}\right) e^{-\lambda \int_{0}^{2 \pi} d \theta e^{X^{0}}\left(e^{i \theta}\right)}\right\rangle_{\text {disk }}^{\mathrm{BCFT}_{0}} .
\end{aligned}
$$

Once this is true for any level-matched primary of $\mathrm{CFT}^{\text {matter }}$, it follows from the Virasoro gluing conditions that

$$
\left|B_{\Psi}\right\rangle=e^{-\lambda \int_{0}^{2 \pi} d \theta e^{X^{0}}}\left|B_{0}\right\rangle,
$$

where $\left|B_{0}\right\rangle$ is the boundary state of $\mathrm{BCFT}_{0} 12$.

To elucidate the relation between Ishibashi states and Ellwood invariants we can compute the energy momentum tensor of the solution. Following Sen (appendix A of [31]), the energy momentum tensor can be extracted from the general form of a boundary state describing a configuration of branes in flat Minkowski spacetime

$|B\rangle=\int \frac{d^{26} k}{(2 \pi)^{26}}\left[F(k)+\left(A_{\mu \nu}(k)+C_{\mu \nu}(k)\right) \alpha_{-1}^{\mu} \bar{\alpha}_{-1}^{\nu}+B(k)\left(b_{-1} \bar{c}_{-1}+\bar{b}_{-1} c_{-1}\right)+\ldots\right] c_{0}^{+} c_{1} \bar{c}_{1}|0, k\rangle$,

where

$$
\begin{aligned}
& A_{\mu \nu}=A_{\nu \mu}, \\
& C_{\mu \nu}=-C_{\nu \mu} .
\end{aligned}
$$

Using this in the linearized equation of motion of Closed String Field Theory, one finds that the source of the graviton (i.e. the energy-momentum tensor) is given by

$$
T_{\mu \nu}(k)=\frac{1}{2}\left(A_{\mu \nu}(k)+\eta_{\mu \nu} B(k)\right) .
$$

By inspection we find that 13

$$
\begin{aligned}
A^{\mu \nu}(k) & =-\frac{1}{2}\left\langle 0,-k\left|c_{-1} \bar{c}_{-1} \alpha_{1}^{(\mu} \bar{\alpha}_{1}^{\nu)} c_{0}^{-}\right| B\right\rangle, \\
B(k) & =\frac{1}{2}\left\langle 0,-k\left|c_{-1} \bar{c}_{-1} \frac{1}{2}\left(c_{1} \bar{b}_{1}+\bar{c}_{1} b_{1}\right) c_{0}^{-}\right| B\right\rangle .
\end{aligned}
$$

\footnotetext{
${ }^{12}$ In general, such a formula formally defines the boundary state for any kind of perturbation. When the operators inserted on the boundary do not commute, path ordering is needed.

${ }^{13} \mathrm{We}$ use the normalization for the BPZ inner product

$$
\left\langle 0, k\left|c_{-1} c_{0} c_{1} \bar{c}_{-1} \bar{c}_{0} \bar{c}_{1}\right| 0, k^{\prime}\right\rangle=(2 \pi)^{26} \delta\left(k+k^{\prime}\right) .
$$
}


Notice that $B(k)$ is the overlap of the boundary state with the ghost dilaton

$$
\left(c \partial^{2} c-\bar{c} \bar{\partial}^{2} \bar{c}\right) e^{i k \cdot X},
$$

which is not a primary field. This seems to imply that $B(k)$ cannot be computed from an Ellwood invariant. However, using the $b c$-gluing conditions

$$
\left(c_{1}+\bar{c}_{-1}\right)|B\rangle=\left(b_{1}-\bar{b}_{-1}\right)|B\rangle=0,
$$

we find that $B(k)$ is also the overlap with the closed string tachyon, which is a primary field

$$
B(k)=\frac{1}{2}\left\langle 0,-k\left|c_{-1} \bar{c}_{-1} c_{0}^{-}\right| B\right\rangle .
$$

In other words, looking at (3.10), we find, on general grounds

$$
B(k)=-F(k) .
$$

Since we are studying a spatially homogeneous process, only the timelike component $k_{0} \equiv-i q$ of the momentum enters the computation.14 We have

$$
\begin{aligned}
B_{\Psi}(q) & =\frac{1}{2}\left\langle e^{-q X^{0}}\left|c_{-1} \bar{c}_{-1} c_{0}^{-}\right| B_{\Psi}\right\rangle=-2 \pi i\left\langle E\left[\tilde{\mathcal{V}}_{T}\right] \mid \tilde{\Psi}-\tilde{\Psi}_{T V}\right\rangle, \\
\tilde{\mathcal{V}}_{T} & =c \bar{c} e^{-q X^{0}} e^{2 \sqrt{\frac{q^{2}}{4}-1} Y} e^{\frac{2 i \phi}{\sqrt{3}}} .
\end{aligned}
$$

From the previous computation we find

$$
\begin{aligned}
B_{\Psi}(q) & =-f_{\lambda}(q) \operatorname{Vol}_{25}, \\
f_{\lambda}(q) & \equiv\left\langle e^{-\lambda \int_{0}^{2 \pi} d \theta e^{X^{0}}\left(e^{i \theta}\right)} e^{-q X^{0}}(0)\right\rangle_{\text {disk }}^{X^{0}}
\end{aligned}
$$

Notice that $B_{\Psi}(q)$ is just the coefficient of the Ishibashi state $\left.\left|e^{q X^{0}}\right\rangle\right\rangle$ in the boundary state. Continuing with $A_{\Psi}^{i j}(q)$ we have

$$
\begin{aligned}
A_{\Psi}^{i j}(q) & =-\frac{1}{2}\left\langle e^{-q X^{0}}\left|c_{-1} \bar{c}_{-1} \alpha_{1}^{(i} \bar{\alpha}_{1}^{j)} c_{0}^{-}\right| B_{\Psi}\right\rangle=2 \pi i\left\langle E\left[\tilde{\mathcal{V}}^{i j}\right] \mid \tilde{\Psi}-\tilde{\Psi}_{T V}\right\rangle, \\
\tilde{\mathcal{V}}^{i j} & =-2 c \bar{c} \partial X^{(i} \bar{\partial} X^{j)} e^{-q X^{0}} e^{q Y} e^{\frac{2 i \phi}{\sqrt{3}}}
\end{aligned}
$$

Computing the Ellwood invariant gives

$$
A_{\Psi}^{i j}(q)=-f_{\lambda}(q) \delta^{i j} \operatorname{Vol}_{25},
$$

\footnotetext{
${ }^{14}$ We define $\left|e^{q X^{0}}\right\rangle \equiv|0,-i q\rangle=\left|0, k_{0}\right\rangle$ and we mimic the needed Wick rotation in time by setting $\left\langle e^{q X^{0}} \mid e^{q^{\prime} X^{0}}\right\rangle^{X^{0}}=2 \pi \delta\left(q+q^{\prime}\right)$.
} 
and trivially also

$$
A_{\Psi}^{i 0}(q)=0 .
$$

It is less straightforward to compute $A_{\Psi}^{00}$. To get this contribution we have to contract the boundary state with the closed string state $W=-2: \partial X^{0} \bar{\partial} X^{0} e^{-q X^{0}}:$. Due to normal ordering this is not a primary field (for nonzero momentum) and we cannot directly compute this contribution from the Ellwood invariant. We have to first decompose the state in primaries and descendants, and use the Virasoro gluing conditions for the boundary state to reexpress the contribution from descendants in terms of primaries

$$
\left\langle e^{-q X^{0}}\right| c_{-1} \bar{c}_{-1} \alpha_{1}^{0} \bar{\alpha}_{1}^{0}=-\frac{2}{q^{2}}\left\langle e^{-q X^{0}}\right| c_{-1} \bar{c}_{-1} L_{1}^{\text {matter }} \bar{L}_{1}^{\text {matter }}, \quad q \neq 0 .
$$

For nonzero momentum we thus have

$$
\begin{aligned}
A_{\Psi}^{00}(q) & =-\frac{1}{2}\left\langle e^{-q X^{0}}\left|c_{-1} \bar{c}_{-1} \alpha_{1}^{0} \bar{\alpha}_{1}^{0} c_{0}^{-}\right| B_{\Psi}\right\rangle \\
& =\frac{1}{2} \frac{2}{q^{2}}\left\langle e^{-q X^{0}}\left|c_{-1} \bar{c}_{-1} L_{1}^{\text {matter }} \bar{L}_{1}^{\text {matter }} c_{0}^{-}\right| B_{\Psi}\right\rangle \\
& =\frac{1}{q^{2}}\left\langle e^{-q X^{0}}\left|c_{-1} \bar{c}_{-1}\left[L_{1}^{\text {matter }}, L_{-1}^{\text {matter }}\right] c_{0}^{-}\right| B_{\Psi}\right\rangle \\
& =\frac{1}{q^{2}} 2 \frac{q^{2}}{4}\left\langle e^{-q X^{0}}\left|c_{-1} \bar{c}_{-1} c_{0}^{-}\right| B_{\Psi}\right\rangle \\
& =B_{\Psi}(q)=-f_{\lambda}(q) \operatorname{Vol}_{25}, \quad q \neq 0 .
\end{aligned}
$$

Notice that this contribution comes from the first nontrivial level of the Ishibashi state

$$
\left.\left|e^{q X^{0}}\right\rangle\right\rangle=\left(1+\frac{1}{2 h} L_{-1}^{\text {matter }} \bar{L}_{-1}^{\text {matter }}+\ldots\right)\left|e^{q X^{0}}\right\rangle,
$$

thus it is not surprising that we get the same result as if we contracted the boundary state with the closed string tachyon.

For $q=0, \partial X^{0} \bar{\partial} X^{0} e^{-q X^{0}}$ is a primary and probes the Ishibashi state $\left.\left|\partial X^{0} \bar{\partial} X^{0}\right\rangle\right\rangle$. Thus for zero momentum we have

$$
\begin{aligned}
A^{00}(q=0) & =-\frac{1}{2}\left\langle 0\left|c_{-1} \bar{c}_{-1} \alpha_{1}^{0} \bar{\alpha}_{1}^{0} c_{0}^{-}\right| B_{\Psi}\right\rangle=2 \pi i\left\langle E\left[\mathcal{V}^{00}\right] \mid \Psi-\Psi_{T V}\right\rangle, \\
\mathcal{V}^{00} & =-2 c \bar{c} \partial X^{0} \bar{\partial} X^{0},
\end{aligned}
$$

which gives

$$
\begin{aligned}
A^{00}(q=0) & =\operatorname{Vol}_{25}\left\langle e^{-\lambda \int_{0}^{2 \pi} d \theta e^{X^{0}}\left(e^{i \theta}\right)}(-2) \partial X^{0} \bar{\partial} X^{0}(0)\right\rangle_{\text {disk }}^{X^{0}} \\
& =\operatorname{Vol}_{26} .
\end{aligned}
$$


Notice that because of momentum conservation the boundary interaction is not giving any contribution, this would not be the case for the cosh $X^{0}$ deformation. The energy momentum tensor in the $q$-space is thus given by

$$
\begin{aligned}
T_{\Psi}^{i j}(q) & =\frac{1}{2}\left(A_{\Psi}^{i j}(q)+\delta^{i j} B_{\Psi}(q)\right)=-f_{\lambda}(q) \delta^{i j} \mathrm{Vol}_{25}, \\
T_{\Psi}^{i 0}(q) & =0 \\
T_{\Psi}^{00}(q) & =\frac{1}{2}\left(A_{\Psi}^{00}(q)+\eta^{00} B_{\Psi}(q)\right)=0, \quad q \neq 0 \\
T_{\Psi}^{00}(q=0) & =\frac{1}{2}\left(A_{\Psi}^{00}(0)+\eta^{00} B_{\Psi}(0)\right)=\frac{1}{2}\left[\mathrm{Vol}_{26}+f_{\lambda}(0) \mathrm{Vol}_{25}\right] .
\end{aligned}
$$

Looking at the definition of $f_{\lambda}$ we see that

$$
f_{\lambda}(0)=\left\langle e^{-\lambda \int e^{X^{0}}}\right\rangle_{\text {disk }}^{X^{0}}=\langle 1\rangle_{\text {disk }}^{X^{0}}=\operatorname{Vol}_{X^{0}}
$$

since our zero-mode normalization is such that

$$
\mathrm{Vol}_{X^{0}}=\langle 0 \mid 0\rangle=2 \pi \delta(0)
$$

So in total we find

$$
T_{\Psi}^{00}(q)=2 \pi \delta(q) \mathrm{Vol}_{25}
$$

It remains to compute the disk amplitude $f_{\lambda}(q)$. In fact, this amplitude has been computed by Larsen et al. in [32]. Here our approach is seemingly different but equivalent: instead of getting time dependence by isolating the time zero mode from the path integral, we contract with the state $e^{-q X^{0}}$ and then Laplace transform in $q$ to the 'closed string time' $x^{0}$, as we discuss later. Combining the results of [32] with the appropriate momentum conservation, we get

$$
\begin{aligned}
& f_{\lambda}(q)=\left\langle e^{-\lambda \int_{0}^{2 \pi} d \theta e^{X^{0}}\left(e^{i \theta}\right)} e^{-q X^{0}}(0)\right\rangle_{\text {disk }}^{X^{0}} \\
& =\sum_{n=0}^{\infty}(-2 \pi \lambda)^{n} 2 \pi \delta(n-q),
\end{aligned}
$$

where we took advantage of the disk geometry and the fact that the distance between the boundary insertions and the bulk insertion is always 1 .

To find the explicit dependence in time we should in principle Wick rotate, Fourier transform and Wick-rotate back. A tailor-made shortcut for this particular example is just to Laplace transform in real time, without any Wick rotation. In particular we have

$$
f_{\lambda}\left(x^{0}\right) \equiv \int_{0}^{\infty} \frac{d q}{2 \pi} f_{\lambda}(q) e^{q X^{0}}=\frac{1}{1+2 \pi \lambda e^{x^{0}}} .
$$


We then find

$$
\begin{aligned}
\frac{T^{i j}\left(x^{0}\right)}{\operatorname{Vol}_{25}} & =-\frac{1}{1+2 \pi \lambda e^{x^{0}}} \delta^{i j}, \\
\frac{T^{00}\left(x^{0}\right)}{\operatorname{Vol}_{25}} & =1 .
\end{aligned}
$$

This is the usual energy-momentum tensor for a half S-brane exhibiting energy conservation and exponential decay for the pressure 15

${ }^{15}$ We noticed the following curiosity: if we change the boundary condition on $X^{0}$ to Dirichlet $X^{0}(0, \pi)=$ $x^{0}$ then (3.1) is no more a solution, because the boundary operator $e^{X^{0}}=e^{x^{0}}$ is now a weight zero number. This off-shell string field is however a state in the $K B c$ algebra and we can easily compute its Ellwood invariant with a graviton vertex operator $\mathcal{V}^{i j}=-2 c \bar{c} \partial X^{i} \bar{\partial} X^{j}$ in quite full generality [17, to find

$$
2 \pi i\left\langle E\left[\mathcal{V}^{i j}\right] \mid \Psi_{\lambda}-\Psi_{T V}\right\rangle^{\left(X^{0} \rightarrow \text { Dir }\right)}=-\frac{1}{1+w \lambda e^{x^{0}}} \delta^{i j} \operatorname{Vol}_{25}=T_{\lambda \rightarrow \frac{w \lambda}{2 \pi}}^{i j}\left(x^{0}\right)=T_{\lambda}^{i j}\left(x^{0}+\log \frac{w}{2 \pi}\right)
$$

where $w \equiv-\left.\frac{d}{d K} F^{2}(K)\right|_{K=0}>0$. Note that although the invariant depends now on the choice of security strip (the 'solution' is no more a solution so changing the security strip is no more a gauge transformation), the dependence is physically irrelevant as it can be absorbed by a redefinition of the marginal parameter and thus removed by a shift in time. This is reminiscent of previous observations on the late time behavior of the rolling tachyon solutions 33,34 . 


\section{Numerical solutions: Lumps in Siegel gauge}

The aim of this section is to show how to construct the boundary state for numerical solutions in the level expansion. Our interest is in the Siegel-gauge lump solutions initially studied in [35, 36, 37, 38] and recently constructed to greater accuracy by two of us (M.K, M.S.) [39].

\subsection{Moeller-Sen-Zwiebach lump at $R=\sqrt{3}$}

The first examples of lower D-branes appearing in string field via inhomogenous tachyon condensation are the tachyon lump solutions found by Moeller, Sen and Zwiebach [35. They construct lump solutions along a compact direction $X$ with radius $R$. Imposing Siegel gauge, twist symmetry and spatial symmetry under reflections $X \rightarrow-X$, such solutions are given up to level $L=3$ in terms of the towers

$$
\begin{aligned}
\left|T_{n}\right\rangle & =c_{1} \cos \left(\frac{n}{R} X(0)\right)|0\rangle, \\
\left|U_{n}\right\rangle & =c_{-1} \cos \left(\frac{n}{R} X(0)\right)|0\rangle, \\
\left|V_{n}\right\rangle & =c_{1} L_{-2}^{(X)} \cos \left(\frac{n}{R} X(0)\right)|0\rangle, \\
\left|W_{n}\right\rangle & =c_{1} L_{-2}^{\prime} \cos \left(\frac{n}{R} X(0)\right)|0\rangle, \\
\left|Z_{n}\right\rangle & =c_{1} L_{-1}^{(X)} L_{-1}^{(X)} \cos \left(\frac{n}{R} X(0)\right)|0\rangle,
\end{aligned}
$$

in the form

$$
|\Psi\rangle=\sum_{n \mid L \leq 3}\left(t_{n}\left|T_{n}\right\rangle+u_{n}\left|U_{n}\right\rangle+v_{n}\left|V_{n}\right\rangle+w_{n}\left|W_{n}\right\rangle+z_{n}\left|Z_{n}\right\rangle\right) .
$$

The Virasoro generators appearing in the expansion of the solution are purely matter, and are split according to the decomposition of the energy momentum tensor

$$
T_{c=26}^{\text {matter }}(z)=T_{c=1}^{(X)}(z)+T_{c=25}^{\prime}(z),
$$

in the two BCFT sectors

$$
\mathrm{BCFT}_{c=26}^{\text {matter }}=\mathrm{BCFT}_{c=1}^{X} \otimes \mathrm{BCFT}_{c=25}^{\prime} .
$$

Ghost degrees of freedom are spanned by ghost oscillators. More zero momentum primaries of $\mathrm{BCFT}^{X}$ appear at higher levels and a more convenient basis is thus given by oscillators in the $X$-direction, [37, 39]. For the time being we consider lump solution 
of the form (4.2) at radius $R=\sqrt{3}$. For this particular value, the reader can find the numerical results for the lump coefficients $\left(t_{n}, u_{n}, v_{n}, w_{n}, z_{n}\right)$ in table 3 of [35].

Our aim is to use the result we derived in section 2.3, which allows us to define the boundary state in terms of the primaries that are switched on in the solution. The formulas (2.66) and (2.72) provide a linear expression for the coefficients of the Ishibashi states in terms of the coefficients of the solution. We will be interested especially in computing the energy density profile of the lump and its pressure along the direction $X$ on which the lump is forming. These quantities can be easily obtained from generalized Ellwood invariants.

What is needed is a lift for the numerical level-truncated solution. Since the solution is not turning on any primary along $\mathrm{BCFT}_{c=25}^{\prime}$, a simple lift is given by (4.2), with the replacement

$$
L^{\prime} \rightarrow L^{\prime}+L^{\text {aux }}
$$

Equivalently, instead of tensoring with an auxiliary BCFT of $c=0$, we can just impose the Dirichlet boundary condition on an arbitrary space direction in $\mathrm{BCFT}_{c=25}^{\prime}$, say $Y \equiv X^{25}$, along which the solution does not change. Because of the universal structure in the $Y$ direction, the solution remains a solution and the coefficients of the Ishibashi states we compute are not affected by the new Dirichlet boundary conditions.

For the energy profile we have to compute the following generalized Ellwood invariants

$$
E_{n} \equiv-4 \pi i\left\langle E\left[c \bar{c} \partial X^{0} \bar{\partial} X^{0} e^{i \frac{n X}{R}+\frac{n Y}{R}}\right] \mid \Psi-\Psi_{T V}\right\rangle .
$$

The $n=0$ contribution is precisely the mass of the brane configuration, normalized to 1 for a single lower dimensional D-brane; it is the coefficient of the Ishibashi state of the zero momentum graviton in the time-time direction. In terms of the momenta $E_{n}$, the energy density profile can be defined as a simple Fourier series 16

$$
E(x) \equiv T^{00}(x)=\frac{1}{\pi R}\left(\frac{1}{2} E_{0}+\sum_{n=1}^{\infty} E_{n} \cos \frac{n x}{R}\right) .
$$

If a solution describes a lower dimensional brane sitting at $x=0$, its energy density profile should be given by

$$
E(x)=\delta(x)=\frac{1}{\pi R}\left(\frac{1}{2}+\sum_{n=1}^{\infty} \cos \frac{n x}{R}\right) .
$$

\footnotetext{
${ }^{16}$ This is $T^{00}=\frac{1}{2}\left(A^{00}-B\right)$ as defined in (3.13). In the present context, it is easy to check that $A^{00}=-B=E$, see (3.29). In this section we set the volume of $\mathrm{CFT}^{\prime}$ to unity. Moreover, we normalize the zero mode in the $X$-CFT by setting $\langle 0 \mid 0\rangle=R$. With this choice the observables we compute are naturally related to integer numbers.
} 
Thus an exact lump solution sitting at $x=0$ will be characterized by

$$
E_{n}=1, \quad \forall n=0, \ldots, \infty \quad \text { (Exact Lump). }
$$

To compute the pressure we need in addition the coefficient of the Ishibashi state for a zero momentum graviton along the $X$-direction, which is captured by the Ellwood invariant

$$
D \equiv 4 \pi i\left\langle E[c \bar{c} \partial X \bar{\partial} X] \mid \Psi-\Psi_{T V}\right\rangle .
$$

This quantity measures how much the original Neumann boundary conditions on the $X$ BCFT are changed to Dirichlet by the solution. If the solution describes the perturbative vacuum with Neumann boundary conditions we will have

$$
D^{N}=+R \sim\langle\partial X \bar{\partial} X(0)\rangle_{\text {disk }}^{\text {Neumann }},
$$

while for a single lump we should have

$$
D^{D}=-1 \sim\langle\partial X \bar{\partial} X(0)\rangle_{\text {disk }}^{\text {Dirichlet }} .
$$

While the non-constant Fourier modes of the pressure transverse to the lump are trivially zero, 17 its constant mode is given by

$$
P \equiv-\frac{1}{2}\left(D+E_{0}\right) .
$$

For the perturbative vacuum, both quantities add up with the same sign giving $P=-E_{0}$, but for the exact lump solution they should cancel each other

$$
P=0 \quad \text { (Exact Lump), }
$$

i.e. the pressure transverse to a D-brane is zero.

Let's see how to compute the $E_{n}$ 's and $D$. In order to do so, it is very convenient to use the conservation laws for the Ellwood invariant. These conservation laws have been first derived in [7] and [27]. In appendix B we offer more streamlined derivation and we use it to write down few more such laws that are essential for the study of lumps. The gauge invariant combinations $E_{n}$ and $D$ can be obtained from Ellwood invariants associated to weight-zero closed-string primary vertex operators

$$
\mathcal{V}^{h}=c \bar{c} V_{\mathrm{CFT}^{\prime}}^{(1-h)} V_{\mathrm{CFT}_{X}}^{(h)}
$$

\footnotetext{
${ }^{17}$ The pressure itself is defined as $T^{X X}=\frac{1}{2}\left(A^{X X}+B\right)=\frac{1}{2}\left(A^{X X}-A^{00}\right)$, see the discussion around (3.13). Its computation proceeds analogously to the $T^{00}$ computed in the previous section for the rolling tachyon, see (3.28). The nonzero momentum part of $A^{X X}$ is computed by writing $\partial X \bar{\partial} X e^{i n X / R}$ as a descendant of the tachyon $e^{i n X / R}$, which precisely cancel the corresponding contribution from $B=-A^{00}$. So only the zero momentum part is nontrivial and it gives rise to the expression (4.11).
} 
with weight $(h, h)$ in the $X$-sector. Applying the conservation laws we find

$$
\begin{aligned}
\left\langle E\left[\mathcal{V}^{h}\right] \mid U_{n}\right\rangle & =\left\langle E\left[\mathcal{V}^{h}\right] \mid T_{n}\right\rangle, \\
\left\langle E\left[\mathcal{V}^{h}\right] \mid V_{n}\right\rangle & =-\left(16 h-\frac{1}{2}\right)\left\langle E\left[\mathcal{V}^{h}\right] \mid T_{n}\right\rangle, \\
\left\langle E\left[\mathcal{V}^{h}\right] \mid W_{n}\right\rangle & =\left(16 h-\frac{7}{2}\right)\left\langle E\left[\mathcal{V}^{h}\right] \mid T_{n}\right\rangle, \\
\left\langle E\left[\mathcal{V}^{h}\right] \mid Z_{n}\right\rangle & =-2 \frac{n^{2}}{R^{2}}\left\langle E\left[\mathcal{V}^{h}\right] \mid T_{n}\right\rangle .
\end{aligned}
$$

Thus, up to level 3

$$
\begin{aligned}
E_{n} & =-4 \pi i\left\langle E\left[c \bar{c} \partial X^{0} \bar{\partial} X^{0} e^{i \frac{n X}{R}+\frac{n Y}{R}}\right] \mid \Psi-\Psi_{T V}\right\rangle \\
& =-4 \pi i \sum_{m \mid L \leq 3} f_{n m}\left\langle E\left[c \bar{c} \partial X^{0} \bar{\partial} X^{0} e^{i \frac{n X}{R}+\frac{n Y}{R}}\right] \mid T_{m}\right\rangle,
\end{aligned}
$$

where the coefficients $f_{n m}$ are given by the conservation laws (4.13)

$$
f_{n m}=-\delta_{m 0} \frac{2}{\pi}+t_{m}+u_{m}-\left(4 \frac{n^{2}}{R^{2}}-\frac{1}{2}\right) v_{m}+\left(4 \frac{n^{2}}{R^{2}}-\frac{7}{2}\right) w_{m}-\frac{2 m^{2}}{R^{2}} z_{m} .
$$

Notice that the tachyon vacuum has been subtracted from the solution by the $-\frac{2}{\pi}\left|T_{0}\right\rangle$ term in $f_{n m}$. We are left with a single Ellwood invariant whose computation gives, see (2.72),

$$
-4 \pi i\left\langle E\left[c \bar{c} \partial X^{0} \bar{\partial} X^{0} e^{i \frac{n X}{R}+\frac{n Y}{R}}\right] \mid T_{m}\right\rangle=-\frac{\pi R}{2} 4^{\frac{n^{2}}{R^{2}}} \delta_{n m} \frac{1+\delta_{n 0}}{2} .
$$

The last factor came from the overlap of the cosine mode with the exponential momentum mode in the closed string vertex operator. In the end we thus find

$$
E_{n}=-\frac{\pi R}{2} 4^{\frac{n^{2}}{R^{2}}} \frac{1+\delta_{n 0}}{2} f_{n n}
$$

The computation of $D$ proceeds analogously but in a simpler way, since only the zero momentum part of the solution participates. Using the conservation laws (4.13) we find

$$
\begin{aligned}
D & =4 \pi i\left\langle E[c \bar{c} \partial X \bar{\partial} X] \mid \Psi-\Psi_{T V}\right\rangle \\
& =4 \pi i d_{0}\left\langle E[c \bar{c} \partial X \bar{\partial} X] \mid T_{0}\right\rangle \\
& =-\frac{\pi R}{2} d_{0},
\end{aligned}
$$

where, up to $L=3$

$$
d_{0}=-\frac{2}{\pi}+t_{0}+u_{0}-\frac{31}{2} v_{0}+\frac{25}{2} w_{0}
$$

Using the coefficients given in table 3 of [35] we find the following values 

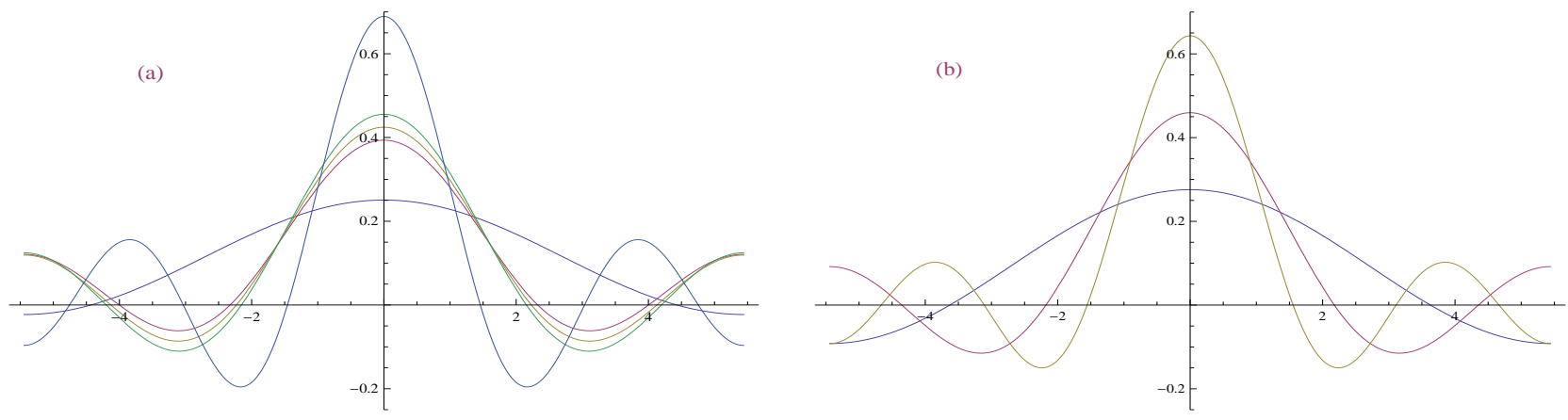

Figure 4.1: (a) Gauge-invariant energy density profile of the Siegel-gauge-lump at $R=\sqrt{3}$, at levels $L=\frac{1}{3}, \frac{4}{3}, 2, \frac{7}{3}, 3$, as defined by eq. (4.5). At level $1 / 3$ only one harmonic is available and corresponds to the less localized profile. At levels $L=4 / 3,2,7 / 3$ the second harmonic enters the game and the profile is essentially unchanged till $L=3$ where the third harmonic gives a substantial contribution. (b) Plot of $\frac{1}{\pi R}\left(\frac{1}{2}+\sum_{n=1}^{N} \cos \frac{n}{R} x\right)$, for $N=1,2,3$, at $R=\sqrt{3}$. This is how the delta-function forms in an expansion in harmonics.

\begin{tabular}{|c|c|cccc|c|c|}
\hline$L(R=\sqrt{3})$ & Action & $E_{0}$ & $E_{1}$ & $E_{2}$ & $E_{3}$ & $D$ & $P$ \\
\hline $1 / 3$ & 1.32002 & 1.23951 & 0.743681 & - & - & 1.23951 & -1.23951 \\
\hline $4 / 3$ & 1.25373 & 1.14776 & 0.741903 & 0.825738 & - & 1.14776 & -1.14776 \\
\hline 2 & 1.11278 & 1.10298 & 0.830459 & 0.927894 & - & -0.574734 & -0.264122 \\
\hline $7 / 3$ & 1.07358 & 1.07489 & 0.899585 & 1.0405 & - & -0.992768 & -0.0410632 \\
\hline 3 & 1.06421 & 1.0645 & 0.89973 & 1.07981 & 1.23776 & -1.08289 & 0.00919196 \\
\hline Expected & 1 & 1 & 1 & 1 & 1 & -1 & 0 \\
\hline
\end{tabular}

In the first column we have also written down the mass of the lump as computed from the action, see column $r^{(1)}$ in table 4 of [35]. The pressure is nicely going to zero. To give an optical visualization we plot the energy density profile in figure 4.17. To compare we plot the approximants of the delta function $\frac{1}{\pi R}\left(\frac{1}{2}+\sum_{n=1}^{N} \cos \frac{n}{R} x\right)$ for $N=1,2,3$, see figure $4.1 \mathrm{~b}$. It is also interesting to qualitatively compare with the known open-string-tachyon profile (given by $\sum_{n} t_{n} \cos \frac{n}{R} x$, see figure 4.2). It is apparent that in the 'closed-string' profile of figure 4.1 the higher harmonics play an essential role in localizing it to zero width, while this does not happen in the open string profile. This is a consequence of the geometry of the identity string field, which effectively dresses the tachyon coefficients $t_{n}$ with $4 \frac{n^{2}}{R^{2}}$ thus amplifying the effect of higher harmonics. As it often happens, subleading contributions in the Fock space can have important sizable effects in observables.

Up to here, we have just used the coefficients given in [35]. There, the maximum level reached was $L=3$, which allowed us to prove our assertions about the energy profile 


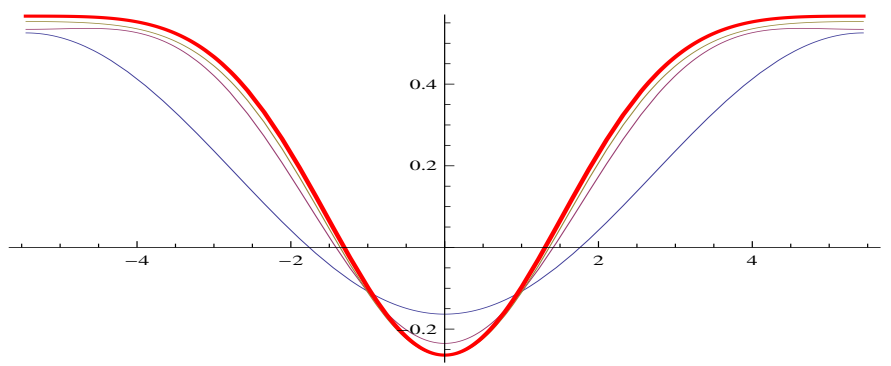

Figure 4.2: Traditional open string tachyon profile of the Siegel-gauge-lump at $R=\sqrt{3}$, at levels $L=\frac{1}{3}$ (blue line), 2 (magenta line), 3 (yellow line) and $L=12$ (red thick line): higher harmonics are suppressed in the Fock space and the open string profile is essentially unchanged as we increase the level.

and the Ellwood invariants with few percent accuracy. Obviously, better accuracy is always desirable, but more fundamentally, one could worry that subtleties related to the identity string field might start manifesting themselves at higher levels. With the code developed by two of the authors [39] it is possible to go up to $L=10$ with a reasonable personal computer power and to explore the lump solutions for different radii. Using cluster facilities we arrived to level 12 and, for $R=\sqrt{3}$ in the same $(L, 2 L)$ scheme as 35], we found 18

\begin{tabular}{|c|c|ccccccc|c|}
\hline$L$ & Action & $E_{0}$ & $E_{1}$ & $E_{2}$ & $E_{3}$ & $E_{4}$ & $E_{5}$ & $E_{6}$ & $D$ \\
\hline 1 & 1.32002 & 1.23951 & 0.74368 & - & - & - & - & - & 1.23951 \\
\hline 2 & 1.11278 & 1.10298 & 0.83046 & 0.927897 & - & - & - & - & -0.574733 \\
\hline 3 & 1.06421 & 1.0645 & 0.899731 & 1.07981 & 1.23776 & - & - & - & -1.08289 \\
\hline 4 & 1.03731 & 1.04598 & 0.917185 & 0.940436 & 1.3942 & - & - & - & -0.841392 \\
\hline 5 & 1.03006 & 1.04024 & 0.939622 & 0.935288 & 0.7325 & - & - & - & -0.782913 \\
\hline 6 & 1.02141 & 1.02947 & 0.945748 & 0.992221 & 0.688677 & 1.9835 & - & - & -0.932662 \\
\hline 7 & 1.01893 & 1.02694 & 0.956255 & 0.995945 & 1.08921 & 2.07561 & - & - & -0.955753 \\
\hline 8 & 1.01477 & 1.02304 & 0.959451 & 0.977503 & 1.12074 & -0.232145 & - & - & -0.912786 \\
\hline 9 & 1.01363 & 1.02183 & 0.965378 & 0.977053 & 0.869997 & -0.30867 & 3.5736 & - & -0.91416 \\
\hline 10 & 1.0112 & 1.01792 & 0.96745 & 0.993393 & 0.86486 & 1.9131 & 3.75069 & - & -0.961774 \\
\hline 11 & 1.01058 & 1.01715 & 0.97137 & 0.993749 & 1.04171 & 1.97574 & -3.86516 & - & -0.9657950 \\
\hline 12 & 1.008998 & 1.01550 & 0.97278 & 0.98704 & 1.051325 & 0.023453 & -4.14698 & 8.073065 & -0.94658 \\
\hline Exp. & 1 & 1 & 1 & 1 & 1 & 1 & 1 & 1 & -1 \\
\hline
\end{tabular}

The first three lines reproduce the results obtained before using the coefficients of [35]. All the energy harmonics appearing at $L=3$ show better approximation to the correct

\footnotetext{
${ }^{18} \mathrm{At}$ higher levels it is more convenient to span the state space of $\mathrm{BCFT}^{X}$ with oscillators acting on momentum modes. The conservation laws that are needed to compute the above invariants are derived in appendix B.
} 
value 1. However starting at level 6 the $E_{4}$ harmonic enters the game and it will take some more levels for it to start converging to 1 . Indeed it appears that higher harmonics oscillate quite erratically before converging to the expected value 19 We notice that also the invariant $D$ (which is a genuine Ellwood invariant) is oscillating with a not so clear pattern with a behavior similar to the one observed in [40].

\subsection{Double lumps at $R=2 \sqrt{3}$}

For $R>2$ multiple lump solutions are energetically reachable from the perturbative vacuum via tachyon condensation. As a further application of our formalism we considered double lump solutions. These belong to the family of recently discovered numerical solutions in [39]. The interest here is to show how our gauge invariant expression for the energy density can be used to measure the distance between the lower dimensional branes described by the solution. Another gauge invariant measurement of the distance would be given by the mass of stretched strings between the multiple separated D-branes, which might be harder to measure in the level expansion with enough precision, as it would require a careful study of the linearized fluctuations.

Suppose we have a solution $\Psi_{a}$ describing two D-branes on a circle of radius $R$, symmetric around the origin and at a distance $a(2 \pi R)$ from each other. The energy of the solution will be given by

$$
E_{0}=2,
$$

meaning that we have two lower dimensional branes. But how does the number $a$ show up in the $E_{n}$ 's? The exact profile of a double lump configuration with separation $a(2 \pi R)$, centered around $\pi R$, is given by

$$
E_{(a)}(x)=\delta(x-\pi R(1-a))+\delta(x-\pi R(1+a))=\frac{1}{\pi R}\left(\frac{1}{2} E_{0}+\sum_{n=1}^{\infty} E_{n} \cos \frac{n x}{R}\right) .
$$

${ }^{19}$ The convergence of the $E_{n}$ 's in level truncation does not appear to be uniform and the level at which $E_{n}$ starts converging increases with $n$. Technically speaking, the energy profile must be understood as

$$
E(x) \equiv \frac{1}{\pi R} \lim _{N \rightarrow \infty}\left[\lim _{L \rightarrow \infty}\left(\frac{1}{2} E_{0}^{(L)}+\sum_{n=1}^{N} E_{n}^{(L)} \cos \frac{n x}{R}\right)\right] .
$$

In terms of the geometrical definition of the level expansion this means that one would need to consider

$$
\lim _{z \rightarrow 1} \lim _{L \rightarrow \infty}\left\langle E[V]\left|z^{L_{0}}\right| \Psi^{(L)}\right\rangle,
$$

so that we first let the approximate solution $\Psi^{(L)}$ to converge to the exact solution and then we send the regulating strip to zero width. In this way there is a natural cutoff given by $z^{\frac{n^{2}}{R^{2}}}$ for higher harmonics. 
Integrating both sides against $\cos \frac{x}{R}$ gives

$$
\int_{0}^{2 \pi R} d x \cos \left(\frac{x}{R}\right) E_{(a)}(x)=-2 \cos (\pi a)=E_{1} .
$$

Thus, in the case of a two-lump solution, the invariant $E_{1}$ measures the distance between the two D-branes

$$
a_{1}=\frac{1}{\pi} \arccos \left(-\frac{E_{1}}{2}\right)
$$

The arc-cosine is defined here in the standard branch $\arccos (0)=\frac{\pi}{2}$. The other branches would give the lengths of all the possible open strings stretching between the branes and wrapping the circle at the same time. Higher harmonics can also be used to compute the distance, and integrating (4.21) against $\cos \frac{n x}{R}$ we find

$$
E_{n}=2(-1)^{n} \cos (n \pi a)
$$

Solving this equation for $a$ requires some care in choosing the correct branch of the arccosine. This must be done in such a way that the distance computed from any $E_{n}$ gives the same value $a_{1}$ as computed from $E_{1}$. The result can be written as

$$
a_{n}=(-1)^{p_{n}} \frac{1}{\pi n} \arccos \left((-1)^{n} \frac{E_{n}}{2}\right)+\frac{2\left[\frac{p_{n}+1}{2}\right]}{n}, \quad n>1
$$

where $[x]$ stands for integer part and the integer $p_{n}$ is uniquely chosen such that

$$
\frac{p_{n}}{n}<a_{1}<\frac{p_{n}+1}{n}
$$

which gives

$$
p_{n}=\left[n a_{1}\right]
$$

Clearly for the exact solution $\Psi_{a}$ we should have

$$
a_{n}=a \equiv \text { Distance, } \quad \forall n \geq 1,
$$

which is a quite nontrivial constraint between the various $E_{n}$, which will be only approximatively satisfied at finite level. For generic multiple lump solutions, the relative distances between the various D-branes can be computed from the $E_{n}$ invariants along similar lines. Let us look at a particular example. At level $(12,36)$ we selected a double lump solution obtained at $R=2 \sqrt{3}$ which displays the open string tachyon profile shown in figure 4.3. The gauge invariant data of the solution are given by 20

\footnotetext{
${ }^{20}$ The solutions at level 2 and 4 (marked in the table with an asterisk) are actually complex. We show the real part of the observables (which would contain a tiny imaginary part of order $10^{-5}-10^{-1}$ depending on the observable and the level).
} 


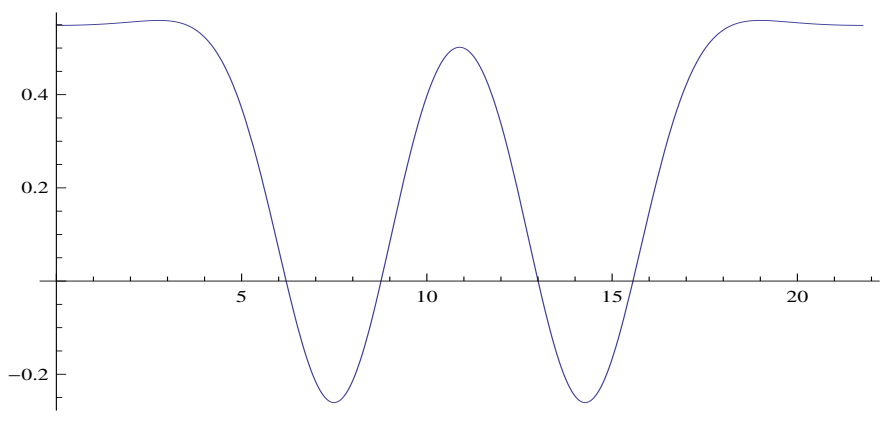

Figure 4.3: Open string tachyon profile of a two-lump solution obtained at $R=2 \sqrt{3}$ and level $L=(12,36)$.

\begin{tabular}{|c|c|c|cccccc|}
\hline$L$ & Action & $D$ & $E_{0}$ & $E_{1}$ & $E_{2}$ & $E_{3}$ & $E_{4}$ & $E_{5}$ \\
\hline 1 & 2.57014 & 2.4209 & 2.4209 & -0.816955 & -0.54184 & 1.3133 & - & - \\
\hline $2^{*}$ & 2.21165 & -1.69337 & 2.1897 & -0.848747 & -0.60583 & 1.89707 & -1.62092 & - \\
\hline 3 & 2.19355 & -2.50001 & 2.11767 & -0.908501 & -0.838798 & 1.84278 & -1.24372 & -0.987367 \\
\hline $4^{*}$ & 2.06874 & -1.39183 & 2.08709 & -0.919667 & -0.850043 & 1.88425 & -1.0523 & -1.02488 \\
\hline 5 & 2.05531 & -1.37542 & 2.07382 & -0.983959 & -0.812633 & 1.91245 & -1.15202 & -0.57724 \\
\hline 6 & 2.03894 & -2.09185 & 2.05368 & -1.00138 & -0.788653 & 1.92175 & -1.30591 & -0.518028 \\
\hline 7 & 2.03494 & -2.1419 & 2.04912 & -1.03283 & -0.765547 & 1.90846 & -1.35827 & -0.488344 \\
\hline 8 & 2.0269 & -1.71527 & 2.04119 & -1.04599 & -0.743696 & 1.90879 & -1.35485 & -0.42022 \\
\hline 9 & 2.02525 & -1.70495 & 2.03899 & -1.06273 & -0.734362 & 1.91644 & -1.37781 & -0.37505 \\
\hline 10 & 2.02052 & -2.07063 & 2.03154 & -1.07229 & -0.717661 & 1.91526 & -1.44161 & -0.329759 \\
\hline 11 & 2.01969 & -2.08504 & 2.03029 & -1.08369 & -0.709787 & 1.90937 & -1.45664 & -0.295048 \\
\hline 12 & 2.01658 & -1.81655 & 2.02687 & -1.09091 & -0.696749 & 1.90744 & -1.45907 & -0.256288 \\
\hline Expected & 2 & -2 & 2 & -1.18 & -0.61 & 1.90 & -1.63 & 0.03 \\
\hline
\end{tabular}

\begin{tabular}{|c|ccccccc|}
\hline$L$ & $E_{6}$ & $E_{7}$ & $E_{8}$ & $E_{9}$ & $E_{10}$ & $E_{11}$ & $E_{12}$ \\
\hline 1 & - & - & - & - & - & - & - \\
\hline $2^{*}$ & - & - & - & - & - & - & - \\
\hline 3 & 2.63667 & - & - & - & - & - & - \\
\hline $4^{*}$ & 2.80995 & - & - & - & - & - & - \\
\hline 5 & 1.3382 & -2.40998 & - & - & - & - & - \\
\hline 6 & 1.32239 & -2.61623 & -0.587783 & - & - & - & - \\
\hline 7 & 2.08367 & -0.516486 & -0.299094 & 4.62486 & - & - & - \\
\hline 8 & 2.07383 & -0.514651 & -0.0446295 & 4.54688 & - & - & - \\
\hline 9 & 1.60158 & -2.29289 & -0.074498 & -2.51466 & -6.95806 & - & - \\
\hline 10 & 1.58451 & -2.37617 & 0.281907 & -2.48429 & -7.22557 & - & - \\
\hline 11 & 1.8759 & -1.0672 & 0.380377 & 5.38459 & 8.09111 & 3.54317 & - \\
\hline 12 & 1.86166 & -1.07601 & -0.0969718 & 5.28465 & 8.37884 & 4.10828 & 9.35208 \\
\hline Expected & 1.60 & -1.92 & 0.67 & 1.13 & -2.00 & 1.23 & 0.55 \\
\hline
\end{tabular}


The $\left(E_{0}, D\right)$ invariants and the action are clearly indicating that we are indeed dealing with a two-lump solution. The expected values for the $E_{n}$ 's have been derived using (4.24) from the distance $a_{*}=0.299 \pm 0.001$ computed later on. In appendix $\mathrm{D}$ another, different two-lump solution and a single-lump solution are shown for the same value of $R=2 \sqrt{3}$; all results have been pushed to level $L=(12,36)$.

From $E_{1}$, at the maximal available level $L=12$, we can compute

$$
a_{1}^{(L=12)}=\frac{1}{\pi} \arccos \left(-\frac{E_{1}^{(12)}}{2}\right)=0.316357 .
$$

Looking at figure 4.3 we see that this is consistent with the distance between the minima of the open string tachyon profile, which, at the same level $L=(12,36)$, is given by

$$
a_{\text {open }}^{(L=12)}=0.310439 .
$$

Also $E_{2}$ and $E_{3}$ give approximate distances which are quite close to $a_{\text {open }}$

$$
\begin{aligned}
& a_{2}^{(L=12)}=\frac{1}{2 \pi} \arccos \left(\frac{E_{2}^{(12)}}{2}\right)=0.306633 \\
& a_{3}^{(L=12)}=\frac{1}{3 \pi} \arccos \left(-\frac{E_{3}^{(12)}}{2}\right)=0.300927 .
\end{aligned}
$$

Going further with the harmonics we have to change the branch of the arc-cosine, according to (4.25)

$$
\begin{aligned}
& a_{4}^{(L=12)}=-\frac{1}{4 \pi} \arccos \left(\frac{E_{4}^{(12)}}{2}\right)+\frac{1}{2}=0.309934, \\
& a_{5}^{(L=12)}=-\frac{1}{5 \pi} \arccos \left(-\frac{E_{5}^{(12)}}{2}\right)+\frac{2}{5}=0.30818, \\
& a_{6}^{(L=12)}=-\frac{1}{6 \pi} \arccos \left(\frac{E_{6}^{(12)}}{2}\right)+\frac{1}{3}=0.313486 .
\end{aligned}
$$

Notice, that by (4.25) the $E_{n}$ 's must be bounded by 2 in absolute value, to be consistent with a two-D-brane interpretation. The $E_{n \geq 6}$ 's already show 'incorrect values' up to level 8 and indeed they would need higher level to start showing a convergence pattern. The set of candidate gauge invariant distances $\left(a_{1}, a_{2}, a_{3}, a_{4}, a_{5}\right)$ is plotted in figure 4.4 for the range of levels $L=2,3, \ldots, 12$. To obtain a prediction on the actual distance between the two D-branes, we have to find a way to extrapolate the observables of the approximate 


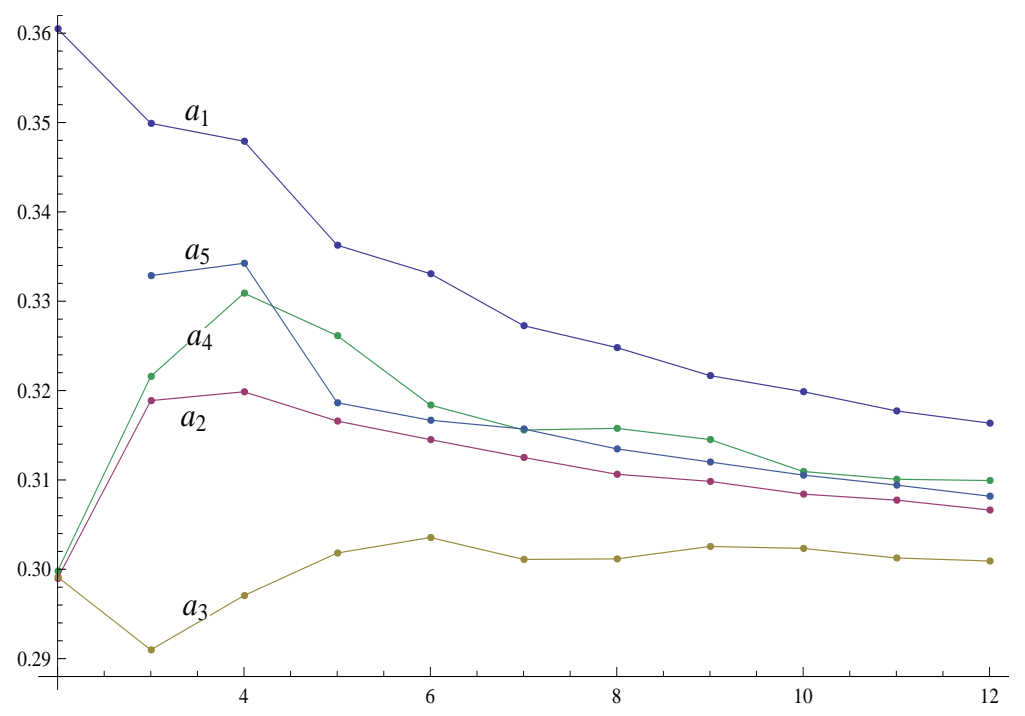

Figure 4.4: Gauge invariant distances $a_{n}^{(L)}$ as computed from the first five $E_{n}$-invariants, as a function of the level $(L=2,3, \ldots, 12)$. Notice that starting from $L=4$ the $a_{n}$ 's show a quite clear (but not so fast) convergence pattern.

level-truncated solution to infinite level. Rather surprisingly, we observed that a simple $1 / L$ fit gives results which are nicely consistent within the first five harmonics. For any harmonic $n=1, \ldots, 5$ we fitted the values of $a_{n}^{(L)}$

$$
a_{n}^{(L)} \approx a_{n}^{\left(\infty, L_{m i n}\right)}+\frac{b_{n}^{\left(\infty, L_{m i n}\right)}}{L},
$$

in the range of levels $\left(L_{\min }, L_{\max }=12\right)$ for all possible $L_{\min }$ 's in the range

$$
4 \leq L_{\min } \leq\left(L_{\max }-2\right)=10 .
$$

The lower bound $4 \leq L_{\min }$ is justified by figure 4.4 and the upper bound is necessary to have at least 3 points to fit. See figure 4.5 for an example.

By varying $L_{\min }$ one can have an estimate of the error of the linear fit. For any frequency $n$ we take the mean value $\bar{a}_{n}^{(\infty)}$ from the results obtained for different $L_{\min }$ and we take the associated standard deviation $\sigma_{n}$ as a measure of the error. The obtained values are the following

\begin{tabular}{|c|c|c|}
\hline$n$ & $\bar{a}_{n}^{(\infty)}$ & $\sigma_{n}$ \\
\hline 1 & 0.300 & 0.001 \\
\hline 2 & 0.299 & 0.001 \\
\hline 3 & 0.299 & 0.004 \\
\hline 4 & 0.299 & 0.003 \\
\hline 5 & 0.298 & 0.002 \\
\hline
\end{tabular}




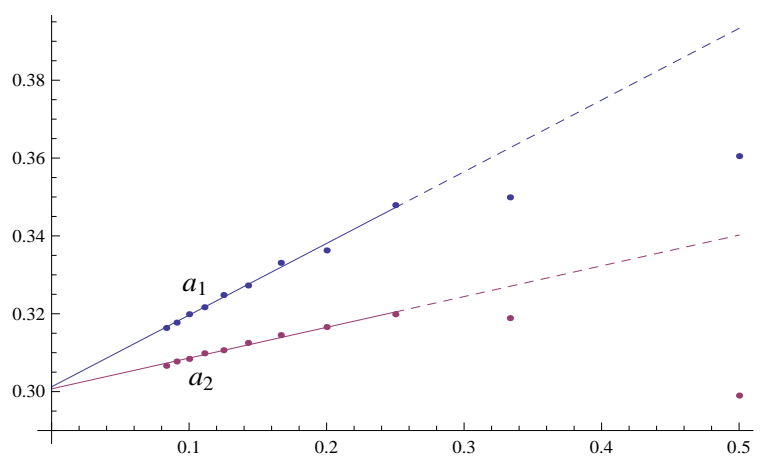

Figure 4.5: Plot of the distances $a_{1}$ and $a_{2}$ as functions of $1 / L$, together with their best fit $a_{n}^{\left(\infty, L_{\min }\right)}+\frac{b_{n}^{\left(\infty, L_{\min }\right)}}{L}$. The fit here is done in the range $L=4, \ldots, 12$, i.e. $L_{\min }=4$, and it appears to essentially capture the dependence of the $a_{n}$ 's with the level.

These are five independent 'measurements' of the distance and the fact that they are all mutually consistent is quite a nontrivial check for the linear fit. Given the mutual consistency between these values, we can average them with weights $w_{n}=1 / \sigma_{n}^{2}$ and obtain the value for the distance

$$
a_{*}=0.299 \pm 0.001,
$$

where the error has been computed with $\left(\sum_{n} w_{n}\right)^{-1 / 2}$.

Since we are 'measuring' a modulus of a BCFT in an unknown point of its moduli space via an approximate OSFT solution, we do not have a given value to compare with, but to appreciate to what extent $a_{*} \sim 0.3$ is consistent with the distance between the two D-branes described by the solution, we plot the energy profile of the solution including up to 6th harmonic against the corresponding truncation of a sum of two delta functions, at distance $a=0.3$, see figure 4.6,

For completeness, few more calculations are needed to completely reconstruct the boundary state for a system of parallel lower dimensional branes. At zero momentum we have an infinite tower of bulk primaries with weight $\left(h^{2}, h^{2}\right)$ for integer $h$. Here we have only considered the coefficients of the Ishibashi states for the identity (captured by $E_{0}$ ) and for the weight $(1,1)$ primary $\partial X \bar{\partial} X$ (captured by $D$ ). We didn't compute the coefficients of the Ishibashi states of the higher level zero momentum primaries. It would also be necessary to verify that the coefficients of the winding-mode Ishibashi states are vanishing, as it must be for Dirichlet boundary conditions. As for the zero momentum primaries, these coefficients too get contribution only from the zero momentum part of the solution. We leave these computations for future work. 

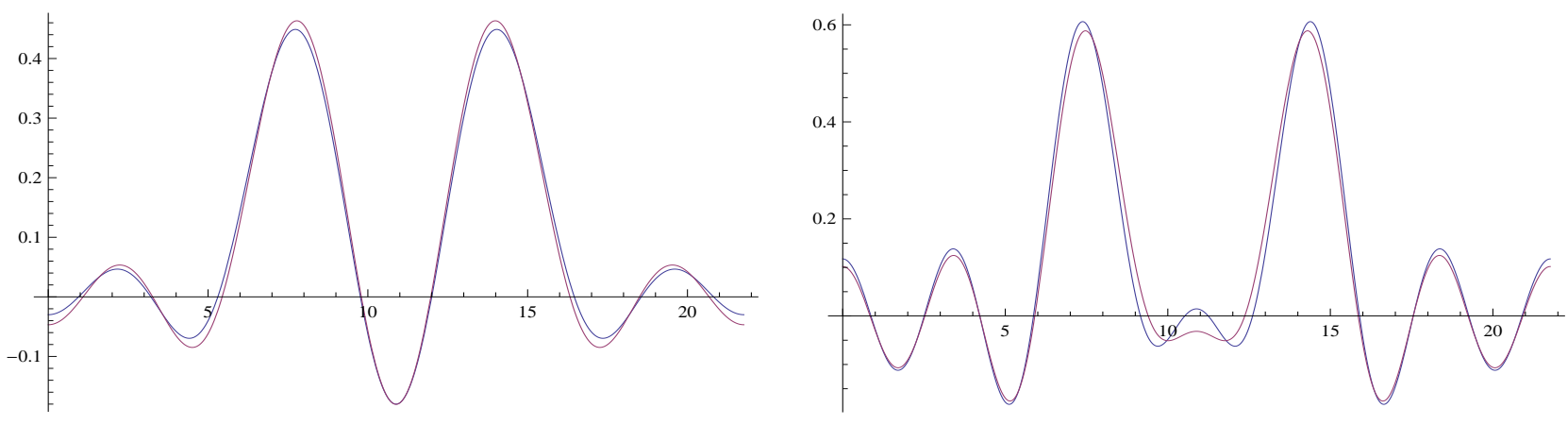

Figure 4.6: Plot of $\frac{1}{\pi R}\left(\frac{1}{2} E_{0}+\sum_{n=1}^{m} E_{n} \cos \frac{n x}{R}\right)$, in blue line, against the corresponding truncation of a sum of two delta functions, in magenta line, at a separation $\bar{a}_{*}=0.3$, for $m=4$ (left) and $m=6$ (right). Here $R=2 \sqrt{3}$ and the coefficients are obtained at level $L=(12,36)$. Notice how the profile of the truncated solution displays a slightly bigger effective distance than the 'exact' one, as if the lumps were getting closer by increasing the level.

\section{Conclusions}

In this paper we have proposed and analyzed a simple shortcut to compute the BCFT boundary state corresponding to a classical solution of OSFT. In a nutshell, OSFT provides the coefficients for the linear combination of Ishibashi states forming the boundary state. These coefficients are given by Ellwood invariants of a lifted solution in a modified OSFT where a trivial BCFT ${ }^{\text {aux }}$ with $c=0$ has been tensored with the original $\mathrm{BCFT}_{0}$. We have characterized the lifting procedure somewhat implicitly, and it would be indeed very interesting to understand it in full generality. In most cases, however, it is sufficient to assign Dirichlet boundary conditions to one spacetime direction. The essence of the trick is to associate to any closed string primary of the form $c \bar{c} V^{\text {matter }}$ a corresponding weight zero primary with nonvanishing tadpole thanks to the Dirichlet boundary condition, without altering the physics described by the solution (the solution remains a solution in the tensor theory). Assuming Ellwood conjecture thus completely defines the boundary state.

Given any solution $\Psi$, a family of closed string states $\left|B_{*}(\Psi)\right\rangle^{(s)}$ has been constructed in [6]. These states are conjectured to be BRST equivalent to the boundary state we deal with in this paper. Among other things, the construction depends on a free parameter $s$ and, in the $s \rightarrow 0$ limit, under some assumptions on the regularity of the solution, one recovers the worldsheet geometry of the Ellwood invariant. Our construction, where spinless matter primaries are lifted to formal elements of the closed string cohomology in an enlarged CFT, applies to [6] as well. All the derivations of [6] go through in the tensor theory and, inside the (enlarged) closed string cohomology, they clearly agree with 
our general results of section 2, assuming Ellwood conjecture. Thus, in principle, the coefficients of the Ishibashi states (2.51), and hence the full BCFT-boundary state, can be computed as well from

$$
n_{\Psi}^{\alpha}=-\frac{1}{2}\left\langle\tilde{\mathcal{V}}^{\alpha}\left|\left(c_{0}-\bar{c}_{0}\right)\right| B_{*}(\tilde{\Psi})\right\rangle^{(s)}
$$

The construction at finite $s$ is a gauge invariant deformation and regularization of the generalized Ellwood invariant. The final $s$-independence of this deformation is a consequence of closed string linearized gauge invariance (the dependence of $\left|B_{*}(\tilde{\Psi})\right\rangle^{(s)}$ on $s$ is, in general, at most BRST exact) and, ultimately, of the validity of the OSFT equation of motion, [6]. This is an important point, given that there are in general infinite families of string fields sharing the same Ellwood invariants with OSFT solutions.

Defining the boundary state from OSFT can potentially contribute to the development of boundary conformal field theory. The classification of consistent boundary states in a given CFT background is, at present, still an open problem. Our proposal gives a complementary way of determining the coefficients of the Ishibashi states, without having to deal with complicated consistency conditions such as Cardy or various sewing conditions [13. What we have to do, instead, is to solve the OSFT equation of motion. This, at least in principle, is a clear well defined task and we have at our disposal a lot of analytic and numerical tools for progressing in this direction.

From the OSFT point of view, it is important to understand whether the space of classical solutions is bigger or smaller than the space of consistent boundary conditions. Suppose OSFT has more solutions than expected: this, for example, could show up in exotic "non integer" values for the Ellwood invariants (an example of this possibility has been found [41, in the context of cubic super string field theory) and hence our boundary state would violate Cardy conditions. On the other hand, our method could prove useful in the search for solutions associated with generic BCFT's whose boundary state is given. Matching the generalized Ellwood invariants of a to-be-found solution to a target boundary state with given BCFT moduli, will partially constrain the coefficients of the solution and a full solution can in principle be searched for in the level expansion (or in some other regularization) by extremizing the constrained action. Consequently one must verify that the full action is also extremized, along the lines of [42]. However it is not guaranteed, see for example [43], that such a solution would exist for any choice of the BCFT moduli. 


\section{Acknowledgments}

We would like to thank Ted Erler, Matthias Gaberdiel, Yuji Okawa and Cornelius Schmidt-Colinet for useful discussions. The access to computing and storage facilities owned by parties and projects contributing to the Czech National Grid Infrastructure MetaCentrum, provided under the programme "Projects of Large Infrastructure for Research, Development, and Innovations" (LM2010005), and to CERIT-SC computing facilities provided under the programme Center CERIT Scientific Cloud, part of the Operational Program Research and Development for Innovations, reg. no. CZ. 1.05/3.2.00/08.0144 is highly appreciated. This research was supported by the EURYI grant GACR EYI/07/E010 from EUROHORC and ESF.

\section{A Example of BCFT ${ }^{\text {aux }}$}

An explicit example of BCFT ${ }^{\text {aux }}$ is given by tensoring a free boson with Dirichlet boundary condition $(c=1)$ with an appropriate $c=-1$ BCFT. For the first Dirichlet factor it is useful to recall the following one-point function 21

$$
\left\langle: e^{q X}:(z, \bar{z})\right\rangle_{\mathrm{disk}}^{\text {Dirichlet }}=\left(1-|z|^{2}\right)^{\frac{q^{2}}{2}}
$$

where we take $q$ to be a generic complex number. For $q$ imaginary, the vertex operator has positive weight. For $q$ real it is a negative weight primary and it is not normalizable as a free field. However in the presence of Dirichlet boundary conditions, the divergence of the zero mode integration is weaker than the delta function imposed by the boundary condition. To derive (A.1) we start with the Green's function on the disk with Dirichlet boundary conditions

$$
G(z, w)=\langle X(z, \bar{z}) X(w, \bar{w})\rangle_{\text {disk }}^{\text {Dirichlet }}=-\frac{1}{2} \log |z-w|^{2}+\frac{1}{2} \log |1-\bar{z} w|^{2},
$$

where the disk is defined by $|z| \leq 1$. One can easily check that

$$
G\left(e^{i \theta}, w\right)=G\left(z, e^{i \theta}\right)=0,
$$

meaning that the Dirichlet condition $X(|z|=1)=0$ is satisfied. The first term is just the usual Green's function on the complex plane (no boundary) while the second is the effect of the Dirichlet boundary conditions. Only the first (bulk) term in (A.2) should be

\footnotetext{
${ }^{21}$ Here, as in the rest of the paper we set $\alpha^{\prime}=1$. In general one should also include the boundary entropy $g_{X}$ factor on the right hand side, but for the auxiliary sector such a factor drops out of all the computations in this paper.
} 
subtracted for closed string vertex operators. Then the result (A.1) easily follows from usual Wick contractions.

An example of the needed $c=-1$ theory can be taken to be a free boson $\varphi$ with background charge $Q$ with an energy momentum tensor given by

$$
T(z)=-: \partial \varphi \partial \varphi:+i Q \partial^{2} \varphi
$$

The central charge is given by

$$
c=1-6 Q^{2}
$$

so that we have $c=-1$ for $Q=\frac{1}{\sqrt{3}}$. The weights of the exponential primary fields are

$$
h\left[e^{i \alpha \varphi}\right]=\frac{\alpha}{2}\left(\frac{\alpha}{2}-Q\right)=\frac{\alpha}{2}\left(\frac{\alpha}{2}-\frac{1}{\sqrt{3}}\right) .
$$

Notice that, differently from the $Q=0$ case, there are now two operators with vanishing conformal weight, one being the identity operator and the other being

$$
w(z)=e^{\frac{2 i}{\sqrt{3}} \varphi(z)}
$$

which is in general needed to screen the background charge. The boundary conditions in the upper half-plane are of Neumann type

$$
\varphi(z)=\bar{\varphi}(\bar{z}), \quad z=\bar{z}
$$

We normalize $w$ such that the disk correlator is given by

$$
\langle w(z)\rangle_{\mathrm{disk}}=\langle\bar{w}(\bar{z})\rangle_{\mathrm{disk}}=\langle w(\xi)\rangle_{\mathrm{UHP}}=\langle\bar{w}(\bar{\xi})\rangle_{\mathrm{UHP}}=1, \quad \forall z, \xi
$$

Another simple option for BCFT ${ }^{\text {aux }}$ is to choose a product of two free bosons $(c=2)$ with Dirichlet boundary conditions, and the symplectic fermion theory with $c=-2$ constructed in 44].22 The advantage of such a choice is that we do not need the analogue of $w$ to saturate the zero modes on the disk.

\section{B Conservation laws for the Ellwood invariant}

In this appendix we derive a set of useful conservation laws for the Ellwood invariant. Some of them have been derived already in [7] and [27]. We present an alternative simpler derivation.

\footnotetext{
${ }^{22}$ Further details and references are summarized in [45].
} 


\section{B.1 Review of conservation laws of the identity string field}

To start with, it is useful to recall some standard conservation laws for the identity string field $|I\rangle$ (see [10, 46] for review). They will later be modified by the closed string vertex operators inserted at the midpoint in the Ellwood invariant. The first such relation is the anomalous conservation of $K_{n}$, for nonvanishing central charge $c$,

$$
\begin{aligned}
K_{n} & =L_{n}-(-1)^{n} L_{-n}, \\
\langle I| K_{n} & =\frac{c}{8} n\left(i^{n}+(-i)^{n}\right)\langle I| .
\end{aligned}
$$

Another useful conservation law is given by the modes of current $i \partial X$, which reads

$$
\begin{aligned}
A_{n} & =\alpha_{n}+(-1)^{n} \alpha_{-n}, \\
\langle I| A_{n} & =0 .
\end{aligned}
$$

Because of non-anomalous momentum conservation in the $X$-CFT, this conservation law shows no anomaly. Let's come to the ghost sector. From the conservation of $K_{n}$ we can read-off, by analogy, the conservation of $B_{n}$, which is not anomalous $(b(z)$ is a genuine weight two primary)

$$
\begin{aligned}
B_{n} & =b_{n}-(-1)^{n} b_{-n}, \\
\langle I| B_{n} & =0 .
\end{aligned}
$$

There is also an analogous (anomalous) conservation law for the $c$-ghost which reads

$$
\begin{aligned}
C_{n} & =c_{n}+(-1)^{n} c_{-n}, \\
\langle I| C_{2 n} & =-(-1)^{n}\langle I| C_{0}, \\
\langle I| C_{2 n+1} & =-(-1)^{n}\langle I| C_{1} .
\end{aligned}
$$

\section{B.2 Virasoro conservation laws}

In this section we compute general conservation laws for Virasoro generators. Suppose

that the total CFT is the tensor product $\mathrm{CFT}^{(1)} \otimes \mathrm{CFT}^{(2)}$, of two CFT's with central charges $c$ and $-c$ respectively. The energy-momentum tensor decomposes as

$$
T(z)=T^{(1)}(z)+T^{(2)}(z) .
$$

The weight-zero vertex operator entering the Ellwood invariant can be written as

$$
V(z, \bar{z})=V_{(1)}^{(h, \bar{h})} V_{(2)}^{(-h,-\bar{h})}(z, \bar{z})
$$


where $h$ and $\bar{h}$ are the holomorphic and antiholomorphic weights (not necessarily the same) of $V_{(1)}$ with respect to $T^{(1)}$. The corresponding BRST and conformally invariant Ellwood state is given by

$$
\langle E[V]|=\langle I| V_{(1)}^{(h, \bar{h})} V_{(2)}^{(-h,-\bar{h})}(i,-i) .
$$

We start the computation of the conservation law for the modes of $T^{(1)}$, using the anomalous derivation

$$
K_{n}^{(1)}=L_{n}^{(1)}-(-1)^{n} L_{-n}^{(1)}=\oint \frac{d w}{2 \pi i} v_{n}(w) T^{(1)}(w),
$$

where the holomorphic vector field $v_{n}(w)$ is given by

$$
v_{n}(w)=w^{n+1}-(-1)^{n} w^{-n+1} .
$$

Assuming that $V_{(1)}^{(h, \bar{h})}$ factorizes into the holomorphic and antiholomorphic parts, we have

$$
\begin{aligned}
\langle E[V]| K_{n}^{(1)} & =\langle I| V_{(1)}^{(h, \bar{h})} V_{(2)}^{(-h,-\bar{h})}(i,-i) K_{n}^{(1)} \\
& =\oint_{0} \frac{d w}{2 \pi i} v_{n}(w)\langle I| V_{(1)}^{(h)}(i) V_{(1)}^{(\bar{h})}(-i) V_{(2)}^{(-h,-\bar{h})}(i,-i) T^{(1)}(w) .
\end{aligned}
$$

Now, using the formalism of [47, 10], we write $\langle I|=\langle 0| U_{f}$, with

$$
f(w)=\frac{2 w}{1-w^{2}}
$$

being the identity conformal map and we move the operator $U_{f}$ to the right of the other operators

$$
\begin{aligned}
& \oint_{0} \frac{d w}{2 \pi i} v_{n}(w)\langle I| V_{(1)}^{(h)}(i) V_{(1)}^{(\bar{h})}(-i) V_{(2)}^{(-h,-\bar{h})}(i,-i) T^{(1)}(w) \\
= & \oint_{0} \frac{d w}{2 \pi i} v_{n}(w)\langle 0| V_{(1)}^{(h)}(i) V_{(1)}^{(\bar{h})}(-i)\left(\left[f^{\prime}(w)\right]^{2} T^{(1)}(f(w))+\frac{c}{12} S_{f}(w)\right) V_{(2)}^{(-h,-\bar{h})}(i,-i) U_{f} .
\end{aligned}
$$

At this point we notice that the geometry of the identity string field, together with the involved operator insertions at the midpoint, implies that

$$
\oint_{0}=-\frac{1}{2} \oint_{(i,-i)} .
$$

To see this, we start with the simple observation that the integrand has poles only in $(0, \pm i, \infty)$. In particular, note that there is no pole at \pm 1 . The poles at the midpoint $\pm i$ arise from the $T-V$ contractions and from the Schwarzian derivative

$$
S_{f}(w)=\{f(w), w\}=\frac{6}{\left(1+w^{2}\right)^{2}} .
$$


Then we notice

$$
\begin{aligned}
f\left(-\frac{1}{w}\right) & =f(w), \\
f^{\prime}\left(-\frac{1}{w}\right) & =w^{2} f^{\prime}(w), \\
v_{n}\left(-\frac{1}{w}\right) & =\frac{1}{w^{2}} v_{n}(w), \\
S_{f}\left(-\frac{1}{w}\right) & =w^{4} S_{f}(w),
\end{aligned}
$$

from which it follows from contour deformation that

$$
\oint_{0}=\oint_{\infty}=-\frac{1}{2} \oint_{(i,-i)} .
$$

We thus get

$$
\begin{aligned}
\langle E[V]| K_{n}^{(1)}= & -\frac{1}{2} \oint_{(i,-i)} \frac{d w}{2 \pi i} v_{n}(w)\left[f^{\prime}(w)\right]^{2}\langle 0| V_{(1)}^{(h)}(i) V_{(1)}^{(\bar{h})}(-i) T^{(1)}(f(w)) V_{(2)}^{(-h,-\bar{h})} U_{f} \\
& -\frac{c}{24} \oint_{(i,-i)} \frac{d w}{2 \pi i} v_{n}(w) S_{f}(w)\langle 0| V_{(1)}^{(h)}(i) V_{(1)}^{(\bar{h})}(-i) V_{(2)}^{(-h,-\bar{h})}(i,-i) U_{f} \\
= & -\frac{1}{2} \oint_{i} \frac{d w}{2 \pi i} v_{n}(w)\left[f^{\prime}(w)\right]^{2}\langle 0|\left[\frac{h V_{(1)}^{(h)}(i)}{(f(w)-i)^{2}}+\frac{\partial V_{(1)}^{(h)}(i)}{f(w)-i}\right] \bar{V}_{(1)}^{(\bar{h})}(-i) V_{(2)}^{(-h,-\bar{h})}(i,-i) U_{f} \\
- & \frac{1}{2} \oint_{-i} \frac{d w}{2 \pi i} v_{n}(w)\left[f^{\prime}(w)\right]^{2}\langle 0| V_{(1)}^{(h)}(i)\left[\frac{\bar{h} V_{(1)}^{(\bar{h})}(-i)}{(f(w)+i)^{2}}+\frac{\partial V_{(1)}^{(\bar{h})}(-i)}{f(w)+i}\right] V_{(2)}^{(-h,-\bar{h})}(i,-i) U_{f} \\
- & \frac{c}{24} \oint_{(i,-i)} \frac{d w}{2 \pi i} v_{n}(w) S_{f}(w)\langle 0| V_{(1)}^{(h)}(i) V_{(1)}^{(\bar{h})}(-i) V_{(2)}^{(-h,-\bar{h})}(i,-i) U_{f} .
\end{aligned}
$$

It is easy to see that the terms proportional to the non primary operators $\partial V$ give vanishing contribution 23 Everything thus simplifies down to

$$
\begin{aligned}
& =-\frac{1}{2} \oint_{(i,-i)} \frac{d w}{2 \pi i} v_{n}(w)\left[\frac{c}{12} S_{f}(w)+\left[f^{\prime}(w)\right]^{2}\left(\frac{h}{(f(w)-i)^{2}}+\frac{\bar{h}}{(f(w)+i)^{2}}\right)\right]\langle E[V]| \\
& =n\left[i^{n}\left(\frac{c}{8}-4 h\right)+(-i)^{n}\left(\frac{c}{8}-4 \bar{h}\right)\right]\langle E[V]| .
\end{aligned}
$$

\footnotetext{
${ }^{23}$ This would not be the case if we worked in the geometry of the local coordinate $w$ where we would have ended with the singular insertion $\sim v_{n}(i)\langle I| \partial V(i) \sim 0 \times \infty$. In this case one would need to displace the insertion a bit away from the midpoint and send the regulator to zero after taking the residue. This gives a finite net contribution which adds up to the naive contribution from the double pole. In the global coordinate geometry $\tilde{w}=f(w)$ no regularization is needed and the total contribution just comes from the "double pole" $\sim \frac{1}{(f(w) \pm i)^{2}}$. Similar considerations apply to the other conservation laws we discuss next.
} 
Summarizing, we found

$$
\left\langle E\left[V_{(1)} V_{(2)}\right]\right| K_{n}^{(1)}=n\left[i^{n}\left(\frac{c}{8}-4 h\right)+(-i)^{n}\left(\frac{c}{8}-4 \bar{h}\right)\right]\left\langle E\left[V_{(1)} V_{(2)}\right]\right|
$$

The conservation law for $K_{n}^{(2)}$ is simply obtained by changing $c \rightarrow-c$ and $(h, \bar{h}) \rightarrow$ $(-h,-\bar{h})$.

\section{B.3 Oscillator conservation laws}

It is useful to derive the conservation laws for the current $i \sqrt{2} \partial X$ of a free boson. We focus on Ellwood states with two kinds of closed string vertex operators: pure momentum modes and the zero momentum primary $\partial X \bar{\partial} X$. First we compute the conservation laws of $\alpha$ oscillators acting on momentum modes. We define

$$
A_{n}=\alpha_{n}+(-1)^{n} \alpha_{-n}=\oint \frac{d w}{2 \pi i} g_{n}(w) i \sqrt{2} \partial X(z) .
$$

The function $g_{n}(w)$ is defined as

$$
g_{n}(w)=w^{n}+(-1)^{n} w^{-n}
$$

and obeys

$$
g_{n}\left(-\frac{1}{w}\right)=g_{n}(w)
$$

Acting with $A_{n}$ on an Ellwood state of definite momentum 24

$$
\begin{aligned}
\langle I| e^{i k X}(i,-i) V_{(2)}(i,-i) A_{n} & =i \sqrt{2} \oint_{0} \frac{d w}{2 \pi i} g_{n}(w)\langle I| e^{i k X}(i,-i) V_{(2)}(i,-i) \partial X(w) \\
& =i \sqrt{2} \oint_{0} \frac{d w}{2 \pi i} g_{n}(w) f^{\prime}(w)\langle 0| e^{i k X}(i,-i) \partial X(f(w)) V_{(2)}(i,-i) U_{f} .
\end{aligned}
$$

As in the previous section, here again we notice that, because of (B.25), we can substitute

$$
\oint_{0} \rightarrow-\frac{1}{2} \oint_{(i,-i)}
$$

which is a general property of the identity conservation laws we consider (but it would not be true for anomalous currents). Using the OPE

$$
e^{i k X}(i,-i) \partial X(f(w)) \sim-\frac{i k}{2}\left(\frac{1}{f(w)-i}+\frac{1}{f(w)+i}\right) e^{i k X}(i,-i),
$$

\footnotetext{
${ }^{24}$ The plane wave $e^{i k X}$ is supplemented with a primary in a decoupled sector of weight $-\frac{k^{2}}{4}$, which we call $V_{(2)}$.
} 
and taking the residues at the midpoints we are left with the simple result

$$
\langle I| e^{i k X} V_{(2)}(i,-i) A_{n}=-\left(i^{n}+(-i)^{n}\right) \sqrt{2} k\langle I| e^{i k X} V_{(2)}(i,-i) .
$$

In addition, we need the conservation laws for the Ellwood invariant given by the zero-momentum graviton vertex operator $c \bar{c} \partial X \bar{\partial} X$

$$
\begin{aligned}
& \langle I| c \bar{c}(i,-i) \partial X \bar{\partial} X(i,-i) A_{n} \\
= & i \sqrt{2} \oint_{0} \frac{d w}{2 \pi i} g_{n}(w)\langle I| \partial X(i) \partial X(-i) \partial X(w) c \bar{c}(i,-i) \\
= & i \sqrt{2} \oint_{0} \frac{d w}{2 \pi i} g_{n}(w) f^{\prime}(w)\langle 0| \partial X(i) \partial X(-i) \partial X(f(w)) c \bar{c}(i,-i) U_{f} \\
= & i \sqrt{2}\left(-\frac{1}{2}\right) \oint_{(i,-i)} \frac{d w}{2 \pi i} g_{n}(w) f^{\prime}(w)\langle 0| \partial X(i) \partial X(-i) \partial X(f(w)) c \bar{c}(i,-i) U_{f} \\
= & -\frac{i}{\sqrt{2}} \oint_{(i,-i)} \frac{d w}{2 \pi i} g_{n}(w) f^{\prime}(w)\langle 0|\left(-\frac{1}{2} \frac{\partial X(-i)}{(f(w)-i)^{2}}-\frac{1}{2} \frac{\partial X(i)}{(f(w)+i)^{2}}\right) c \bar{c}(i,-i) U_{f} .
\end{aligned}
$$

Taking the residues at the midpoints we get

$$
\langle I| c \bar{c} \partial X \bar{\partial} X(i,-i) A_{n}=\sqrt{2} n^{2}(-i)^{n}\langle 0| c \bar{c}(i,-i)\left(\partial X(i)-(-1)^{n} \partial X(-i)\right) U_{f}
$$

Notice that we cannot take the $U_{f}$ operator back the vacuum because the leftover insertion at the midpoint has overall negative weight. Applying another $A_{m}$ we get rid of the $\partial X$ insertion and we get

$$
\langle I| c \bar{c} \partial X \bar{\partial} X(i,-i) A_{n} A_{m}=-2(n m)^{2} i^{n+m}\left((-1)^{n}+(-1)^{m}\right)\langle 0| c \bar{c}(i,-i) U_{f}
$$

Further applications of $A_{n}$ give trivially zero.

\section{B.4 Ghost conservation laws}

The conservation law for $b_{n}$ oscillators is easily obtained from

$$
\begin{aligned}
\langle I| c(i) c(-i) V^{(1,1)}(i,-i) B_{n} & =\oint_{0} \frac{d w}{2 \pi i} v_{n}(w)\langle I| c(i) c(-i) V^{(1,1)}(i,-i) b(w) \\
& =\oint_{0} \frac{d w}{2 \pi i} v_{n}(w)\left[f^{\prime}(w)\right]^{2}\langle 0| c(i) c(-i) b(f(w)) V^{(1,1)}(i,-i) U_{f} \\
& =-\frac{1}{2} \oint_{(i,-i)} \frac{d w}{2 \pi i} v_{n}(w)\left[f^{\prime}(w)\right]^{2}\langle 0| c(i) c(-i) b(f(w)) V^{(1,1)}(i,-i) U_{f},
\end{aligned}
$$


where we used

$$
\oint_{0}=-\frac{1}{2} \oint_{(i,-i)},
$$

just as we did in the case of energy-momentum tensor.

Performing the midpoint contractions between $b(f(w))$ and $c( \pm i)$, no residue is found since $v_{n}( \pm i)=0$, and we are left simply with

$$
\langle I| c(i) c(-i) V^{(1,1)}(i,-i) B_{n}=0
$$

The $c$ ghost conservation law is just a bit more complicated. What happens here is that the anomalies in the conservation of $C_{n}$ on the identity, (B.8) and (B.9), are killed by the $c \bar{c}(i,-i)$ from the closed string insertion, as we are now going to see

$$
\begin{aligned}
\langle I| c(i) c(-i) V^{(1,1)}(i,-i) C_{n} & =\oint \frac{d w}{2 \pi i} h_{n}(w)\langle I| c(i) c(-i) V^{(1,1)}(i,-i) c(w) \\
& =\oint_{0} \frac{d w}{2 \pi i} h_{n}(w)\left[f^{\prime}(w)\right]^{-1}\langle 0| c(i) c(-i) c(f(w)) V^{(1,1)}(i,-i) U_{f} \\
& =-\frac{1}{2} \oint_{(i,-i)} \frac{d w}{2 \pi i} h_{n}(w)\left[f^{\prime}(w)\right]^{-1}\langle 0| c(i) c(-i) c(f(w)) V^{(1,1)}(i,-i) U_{f} .
\end{aligned}
$$

Here the quadratic differential $h_{n}(w)$ is given by

$$
h_{n}(w)=w^{-2}\left(w^{n}+(-1)^{n} w^{-n}\right),
$$

and obeys

$$
h_{n}\left(-\frac{1}{w}\right)=w^{4} h_{n}(w)
$$

Once more, this property allows us to replace

$$
\oint_{0} \rightarrow-\frac{1}{2} \oint_{(i,-i)}
$$

in going from the second to third line of $(\underline{B .35})$. Computing the residues at the midpoint we are left with

$$
\begin{aligned}
& \langle I| c(i) c(-i) V^{(1,1)}(i,-i) C_{n} \\
= & -i^{n+1}\langle 0| c(i) c(-i)\left(c(i)-(-1)^{n} c(-i)\right) V^{(1,1)}(i,-i) U_{f} .
\end{aligned}
$$

Notice that $C_{n}$ has been localized to a midpoint insertion in the global coordinate. It is then killed by the two c's from the closed string insertion. Thus, differently from the 
pure identity string field, the conservation law of the $c$ ghost on the Ellwood state is not anomalous

$$
\langle I| c(i) c(-i) V^{(1,1)}(i,-i) C_{n}=0
$$

\section{General properties of the boundary state}

In string theory, the boundary state is a ghost-number-three closed string state and it appears as a source term in the closed string field theory action via the coupling

$$
\left\langle B\left|c_{0}^{-}\right| \Phi\right\rangle
$$

to the dynamical closed string field $\Phi$ of total ghost number two. The closed string inner product contains the usual insertion of $c_{0}^{-}=c_{0}-\bar{c}_{0}$. In order to write down a kinetic term for the closed string field, it is necessary to assume the level matching conditions [48]

$$
L_{0}^{-}|\Phi\rangle=b_{0}^{-}|\Phi\rangle=0
$$

where $L_{0}^{-}=L_{0}-\bar{L}_{0}$ and $b_{0}^{-}=b_{0}-\bar{b}_{0}$. It does not appear consistent to include non-levelmatched closed string states and thus we must impose also

$$
L_{0}^{-}|B\rangle=b_{0}^{-}|B\rangle=0
$$

The boundary state is not just a source term in the closed string action but it is also a peculiar state which incarnates the existence of a boundary in $\mathrm{CFT}_{0}$ (on which we define closed string field theory), which preserves conformal invariance. Together with the previous conditions this means

$$
\begin{aligned}
b_{0}^{-}|B\rangle & =0 \\
\left(L_{n}^{\text {tot }}-\bar{L}_{-n}^{\text {tot }}\right)|B\rangle & =0 \\
\left(Q_{g h}-3\right)|B\rangle & =0
\end{aligned}
$$

where

$$
Q_{g h}=\oint \frac{d z}{2 \pi i}(-: b c:)(z)+h . c .
$$

is the total ghost number. 


\section{C.1 Proof of matter ghost factorization}

Commuting (C.3) with (C.4) we learn that

$$
\left(b_{n}-\bar{b}_{-n}\right)|B\rangle=0, \quad \forall n .
$$

The most general state obeying (C.7) can be written in normal ordered form as

$$
|B\rangle=f\left(\left\{b_{-m}\right\},\left\{\bar{b}_{-m}\right\}, c_{0}^{+},[\text {matter }]\right) \exp \left(-\sum_{n=1}^{\infty} \bar{b}_{-n} c_{-n}+b_{-n} \bar{c}_{-n}\right) c_{1} \bar{c}_{1}|0\rangle_{S L(2, C)}
$$

where $f$ is a generic function depending on $b$-ghost creation operators, $c_{0}^{+}$, and generic matter operators. To see that this is the case focus on the dependence on $\left(b_{-n}, \bar{b}_{-n}, c_{-n}, \bar{c}_{-n}\right)$ for fixed $n \geq 1$. Then conditions (C.7) are equivalent to the differential equations

$$
\begin{aligned}
& \left(\partial_{c_{-n}}-\bar{b}_{-n}\right) B\left(c_{-n}, \bar{c}_{-n}, b_{-n}, \bar{b}_{-n}\right)=0 \\
& \left(\partial_{\bar{c}_{-n}}-b_{-n}\right) B\left(c_{-n}, \bar{c}_{-n}, b_{-n}, \bar{b}_{-n}\right)=0
\end{aligned}
$$

whose generic solution is

$$
B\left(c_{-n}, \bar{c}_{-n}, b_{-n}, \bar{b}_{-n}\right)=f\left(b_{-n}, \bar{b}_{-n}\right) \exp \left(-\bar{b}_{-n} c_{-n}-b_{-n} \bar{c}_{-n}\right) .
$$

Repeating this procedure for every $n \geq 1$ and also for $n=0$, we end up with (C.8). Finally, imposing ghost number three, (C.5), we conclude that

$$
f\left(\left\{b_{-n}\right\},\left\{\bar{b}_{-n}\right\}, c_{0}^{+},[\text {matter }]\right)=c_{0}^{+} g([\text { matter }]) .
$$

Thus we have showed that a state obeying (C.3, C.4, C.5) is necessarily matter-ghost factorized

$$
|B\rangle=|B\rangle^{\text {matter }} \otimes\left|B_{g h}\right\rangle,
$$

and the ghost factor $\left|B_{g h}\right\rangle \equiv\left|B_{b c}\right\rangle$ is the usual boundary state of the $b c$-BCFT

$$
\begin{aligned}
& \left(b_{n}-\bar{b}_{-n}\right)\left|B_{b c}\right\rangle=0, \\
& \left(c_{n}+\bar{c}_{-n}\right)\left|B_{b c}\right\rangle=0 .
\end{aligned}
$$

From the total gluing conditions (C.4) we then find that $\left|B_{\Psi}\right\rangle^{\text {matter }}$ obeys the standard gluing conditions of the matter sector

$$
\left(L_{n}^{\text {matter }}-\bar{L}_{-n}^{\text {matter }}\right)|B\rangle^{\text {matter }}=0
$$

and from this it is easy to check that that $|B\rangle$ is also BRST invariant

$$
(Q+\bar{Q})|B\rangle=0
$$


Few other universal gluing conditions follow from here. Let's look at the anomalous gluing of the ghost current

$$
j_{g h}(z)=-: b c:(z)=\sum_{n} j_{n} z^{-n-1}
$$

which, using the gluing conditions (C.14, C.15) reads

$$
\left(j_{n}+\bar{j}_{-n}-3 \delta_{n 0}\right)|B\rangle=0 .
$$

Notice that this is consistent with (C.5), since $Q_{g h}=j_{0}+\bar{j}_{0}$. From here it also follows that the BRST current

$$
j_{B R S T}(z)=\sum_{n} Q_{n} z^{-n-1}
$$

glues non-anomalously at the boundary. Indeed we have that

$$
Q_{n}=\left[Q, j_{n}\right]
$$

and thus from the ghost current gluing condition it follows that

$$
\left(Q_{n}+\bar{Q}_{-n}\right)|B\rangle=0
$$

\section{C.2 Normalization of the ghost boundary state}

Here we fix the normalization of the ghost boundary state from modular invariance. Consider a cylinder $C_{L, T}$ of circumference $L$ and height $T$. We put $\mathrm{BCFT}_{0}$ boundary conditions on the lower and upper boundary of $C_{L, T}$. We are interested in computing the partition function

$$
\langle 1\rangle_{C_{L, T}}
$$

In string theory, this partition function is identically vanishing because the zero modes of the $b, c$ ghosts are not soaked up. On the cylinder there is a zero mode for $c$ associated with the constant conformal Killing vector (CKV) for rotation of the cylinder around its axis. There is also a zero mode for $b$, associated with the constant holomorphic quadratic differential (HQD) which changes the length of the base circumference. Because both the CKV and the HQD are constant we have that

$$
\begin{aligned}
\left\langle b(w) c\left(w^{\prime}\right)\right\rangle_{C_{L, T}} & \equiv Z_{L, T}, \\
\partial_{w} \partial_{w^{\prime}}\left\langle b(w) c\left(w^{\prime}\right)\right\rangle_{C_{L, T}} & =0,
\end{aligned}
$$

see figure C.1. To compute $Z_{L, T}$ we proceed as follows. We first consider a cylinder of 


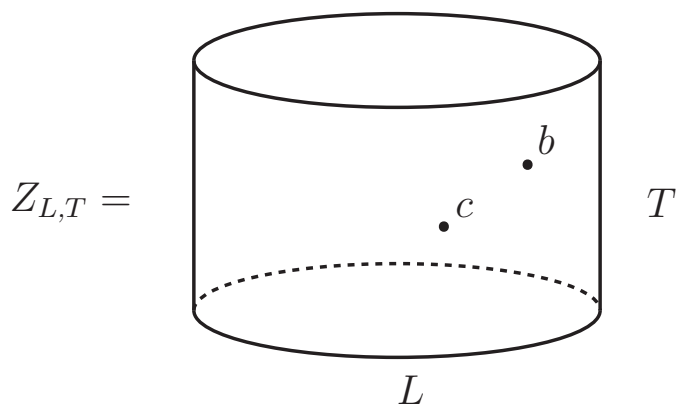

Figure C.1: The partition function $Z_{L, T}$ is given by the path integral on a cylinder $C_{L, T}$, with insertion of $b$ and $c$. The position of the insertions is inessential as only the constant zero modes in the expansion of $b$ and $c$ gives nonvanishing contribution to the path integral.

height $T=\pi$ and circumference $L=2 \pi t$. Every $Z_{L, T}$ can be reduced to $Z_{2 \pi t, \pi}$ by simple scaling, keeping track of the weights of the insertions

$$
\begin{aligned}
Z_{L, T}=\langle b c\rangle_{C_{L, T}} & =\left.\langle f \circ b f \circ c\rangle_{C_{2 \pi t, \pi}}\right|_{t=\frac{L}{2 T}}=\left.\frac{\pi}{T} Z_{2 \pi t, \pi}\right|_{t=\frac{L}{2 T}}, \\
f(w) & =\frac{\pi w}{T} .
\end{aligned}
$$

$Z_{2 \pi t, \pi}$ is just the one loop open string vacuum amplitude (before integration over the moduli space 25

$$
\operatorname{Tr}_{H_{\text {open }}}\left[(-1)^{F} e^{-2 \pi t L_{0}} b_{0} c_{0}\right]=\langle b c\rangle_{C_{2 \pi t, \pi}}=Z_{2 \pi t, \pi} .
$$

This follows from the fact that the cylinder is obtained by identifying the edges of a canonical open string strip of height $\pi$ and length $2 \pi t$. Such a strip is the image of the half annulus in the UHP (defined by $1 \leq|z| \leq e^{2 \pi t}$ and $\Im z \geq 0$ ), obtained by the map

$$
w=\ln z
$$

\footnotetext{
${ }^{25}$ We define the fermion number

$$
F \equiv Q_{g h}-\frac{3}{2}=\int_{0}^{2 \pi i} \frac{d w}{2 \pi i} j_{g h}(w),
$$

as the zero mode of the ghost current in the canonical strip frame (with doubling trick understood). $F$ is anti-hermitian and thus $(-1)^{F}$ is hermitian. The Siegel-gauge projector $b_{0} c_{0}$ is anti-hermitian and the trace we are computing is imaginary.
} 

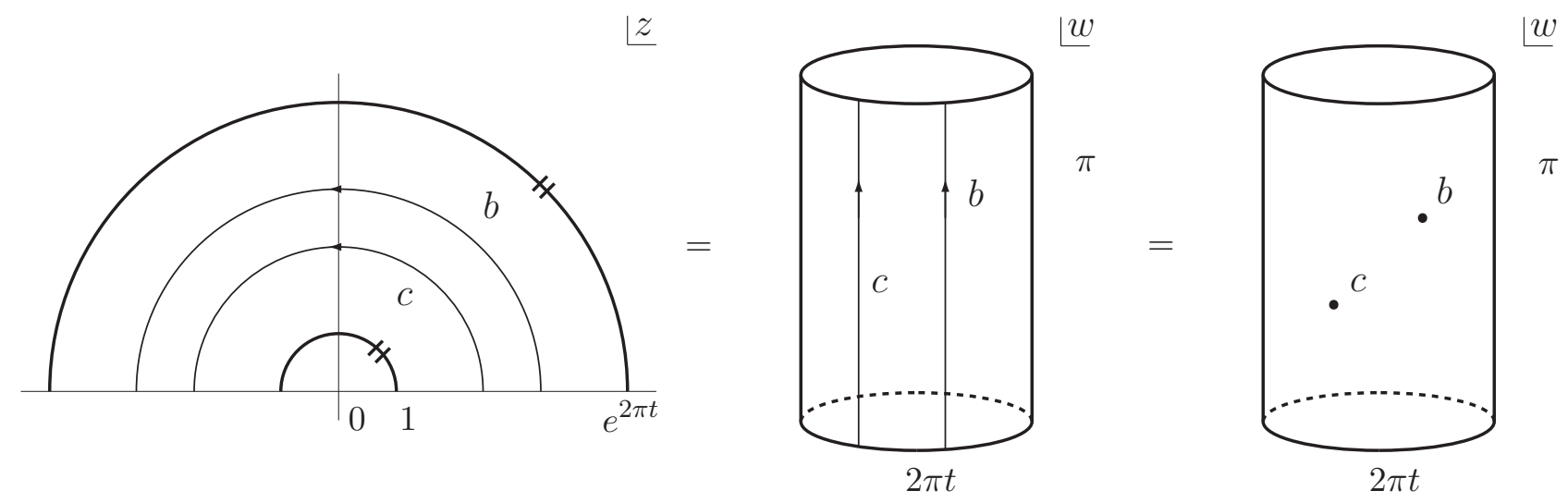

Figure C.2: Open string trace as a path integral on the cylinder, as stated in eq.(C.24). The inner and outer semicircles in the $z$ coordinate are identified by the $\operatorname{Tr}\left[(-1)^{F}(\ldots)\right]$.

The UHP zero modes $b_{0}$ and $c_{0}$ are mapped to vertical line integrals in the $w$ coordinate

$$
\begin{aligned}
& w \circ b_{0}=\oint_{0} \frac{d z}{2 \pi i} z[w \circ b(z)]=\frac{1}{2 \pi} \int_{0}^{2 \pi} d x b(y+i x) \rightarrow b(w), \\
& w \circ c_{0}=\oint_{0} \frac{d z}{2 \pi i} \frac{1}{z^{2}}[w \circ c(z)]=\frac{1}{2 \pi} \int_{0}^{2 \pi} d x c(y+i x) \rightarrow c(w),
\end{aligned}
$$

where we wrote $w=y+i x$ and extended the UHP by the doubling trick to the full complex plane. In the last step we have 'averaged' the integrals because the correlator only gets contribution from the cylinder zero modes which are constant in the $w$ coordinate. This establishes (C.24), see figure C.2

We can equivalently compute $Z_{2 \pi t, \pi}$ by evolving the boundary state $|B\rangle$ with the closed string propagator and contracting with the BPZ dual $\langle B|$. Proceeding similarly to the open string picture, we can write (using the ghost gluing conditions)

$$
\begin{aligned}
\left\langle B\left|e^{-\frac{\pi}{t}\left(L_{0}+\bar{L}_{0}\right)}\left(b_{0}+\bar{b}_{0}\right)\left(c_{0}-\bar{c}_{0}\right)\right| B\right\rangle & =4\left\langle B\left|e^{-\frac{\pi}{t}\left(L_{0}+\bar{L}_{0}\right)} b_{0} c_{0}\right| B\right\rangle \\
& =-4 i\langle b c\rangle_{C_{2 \pi, \frac{\pi}{t}}} \\
& =-4 i Z_{2 \pi, \frac{\pi}{t}}
\end{aligned}
$$

As illustrated in figure C.3, this is easily obtained by mapping the annulus $1 \leq|z| \leq e^{\frac{\pi}{t}}$ to the cylinder $C_{2 \pi, \frac{\pi}{t}}$ with

$$
w=i\left(\frac{\pi}{t}-\log z\right)
$$

and replacing the resulting horizontal line integrals with a local insertion using again that 

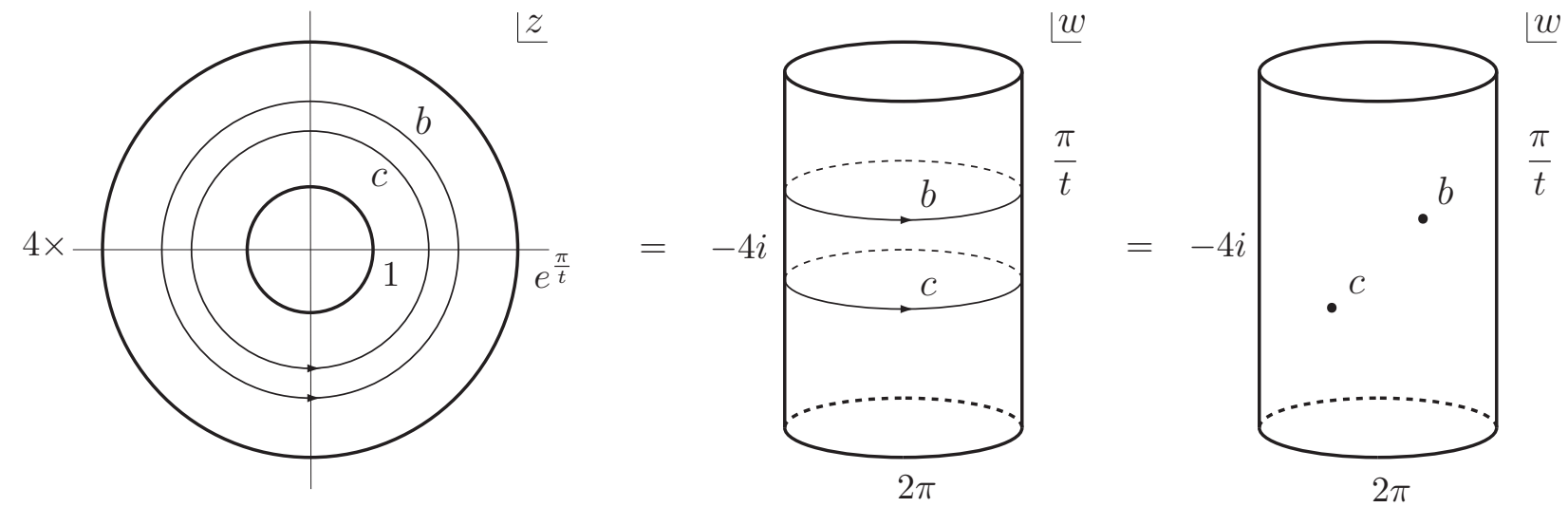

Figure C.3: Graphical representation of eq. (C.27).

the HQD and the CKV for $b$ and $c$ are constant on the cylinder $(w=y+i x)$

$$
\begin{aligned}
& b_{0} \rightarrow \frac{1}{2 \pi} \int_{0}^{2 \pi} d y b(y+i x) \rightarrow b(w), \\
& c_{0} \rightarrow-\frac{i}{2 \pi} \int_{0}^{2 \pi} d y c(y+i x) \rightarrow-i c(w) .
\end{aligned}
$$

Now we use the scaling property (C.23)

$$
Z_{2 \pi, \frac{\pi}{t}}=t Z_{2 \pi t, \pi}
$$

to get

$$
\left\langle B\left|e^{-\frac{\pi}{t}\left(L_{0}+\bar{L}_{0}\right)}\left(b_{0}+\bar{b}_{0}\right)\left(c_{0}-\bar{c}_{0}\right)\right| B\right\rangle=-4 i t \operatorname{Tr}_{H_{\text {open }}}\left[(-1)^{F} e^{-2 \pi t L_{0}} b_{0} c_{0}\right] .
$$

This is an equality between two real quantities. We can use the above equation to determine the overall normalization of the ghost boundary state. A generic boundary state for an open string background is given by

$$
|B\rangle=\left|B_{\text {matter }}\right\rangle \otimes\left|B_{b c}\right\rangle,
$$

where $\left|B_{\text {matter }}\right\rangle$ is the matter boundary state $(c=26)$ obeying Cardy condition

$$
\left\langle B_{\text {matter }}\left|e^{-\frac{\pi}{t}\left(L_{0}^{\text {matter }}+\bar{L}_{0}^{\text {matter }}-\frac{c}{12}\right)}\right| B_{\text {matter }}\right\rangle=\operatorname{Tr}_{H_{\text {open }}^{\text {matter }}}\left[e^{-2 \pi t\left(L_{0}-\frac{c}{24}\right)}\right],
$$

and

$$
\begin{aligned}
& \left|B_{b c}\right\rangle=\mathcal{N}_{g h}\left(c_{0}+\bar{c}_{0}\right) \exp \left(-\sum_{n=1}^{\infty} \bar{b}_{-n} c_{-n}+b_{-n} \bar{c}_{-n}\right) c_{1} \bar{c}_{1}|0\rangle_{S L(2, C)} \\
& \left\langle B_{b c}\right|=-\mathcal{N}_{g h}\langle 0| c_{-1} \bar{c}_{-1} \exp \left(\sum_{n=1}^{\infty} \bar{b}_{n} c_{n}+b_{n} \bar{c}_{n}\right)\left(c_{0}+\bar{c}_{0}\right)
\end{aligned}
$$


are the ghost boundary state and its BPZ dual whose normalization we want to determine. Taking the ghost part of (C.31) and assuming (C.33) we get a Cardy-like condition for the $b c$-BCFT

$$
\left\langle B_{b c}\left|e^{-\frac{\pi}{t}\left(L_{0}+\bar{L}_{0}+\frac{26}{12}\right)}\left(b_{0}+\bar{b}_{0}\right)\left(c_{0}-\bar{c}_{0}\right)\right| B_{b c}\right\rangle=-4 i t \operatorname{Tr}_{H_{\text {open }}^{\text {ghost }}}\left[(-1)^{F} e^{-2 \pi t\left(L_{0}+\frac{26}{24}\right)} b_{0} c_{0}\right] .
$$

Computing the left hand side with (C.34, C.35) we have

$$
\begin{aligned}
\left\langle B_{b c}\left|e^{-\frac{\pi}{t}\left(L_{0}+\bar{L}_{0}+\frac{26}{12}\right)}\left(b_{0}+\bar{b}_{0}\right)\left(c_{0}-\bar{c}_{0}\right)\right| B_{b c}\right\rangle & =-2 \mathcal{N}_{g h}^{2} \eta^{2}\left(\frac{i}{t}\right)\left\langle 0\left|c_{-1} \bar{c}_{-1}\left(c_{0}-\bar{c}_{0}\right)\left(c_{0}+\bar{c}_{0}\right) c_{1} \bar{c}_{1}\right| 0\right\rangle \\
& \left.=-2 \mathcal{N}_{g h}^{2} \eta^{2}\left(\frac{i}{t}\right)(-2)=4 \mathcal{N}_{g h}^{2} \eta^{2}\left(\frac{i}{t}\right), \quad \text { (C. } 37\right)
\end{aligned}
$$

where

$$
\eta(i t)=e^{-\frac{\pi t}{12}} \prod_{n=1}^{\infty}\left(1-e^{-2 \pi n t}\right)
$$

is the Dedekind $\eta$-function and we normalize the ghost BPZ-inner product as 26

$$
\left\langle 0\left|c_{-1} c_{0} c_{1} \bar{c}_{-1} \bar{c}_{0} \bar{c}_{1}\right| 0\right\rangle \equiv 1
$$

Computing the open string trace and using the usual modular property of the Dedekind eta function

$$
\sqrt{t} \eta(i t)=\eta\left(\frac{i}{t}\right)
$$

we find

$$
-4 i t \operatorname{Tr}_{H_{\mathrm{open}}^{\text {ghost }}}\left[(-1)^{F} e^{-2 \pi t\left(L_{0}+\frac{26}{24}\right)} b_{0} c_{0}\right]=4 t \eta^{2}(i t)=4 \eta^{2}\left(\frac{i}{t}\right)
$$

This gives

$$
\mathcal{N}_{g h}^{2}=1
$$

Notice how the scaling law (C.30) accounts for the modular transformation (C.39). We can fix the sign in $\mathcal{N}_{g h}$ by asking

$$
\left\langle B_{b c}\left|\left(c_{0}-\bar{c}_{0}\right)\right| c \bar{c}\right\rangle=\left\langle\left(c_{0}-\bar{c}_{0}\right) c \bar{c}(0)\right\rangle_{\text {disk }} .
$$

\footnotetext{
${ }^{26}$ In the closed string Hilbert space, Hermitian and BPZ conjugation differ by an overall factor of $i$. In our conventions (slightly different from [48])

$$
\mathrm{BPZ}(|0\rangle) \equiv\langle 0|=i_{h c}\left(0 \mid \equiv i(|0\rangle)^{\dagger} .\right.
$$

The basic hermitian inner product is thus given by

$$
{ }_{h c}\left\langle 0\left|c_{-1} c_{0} c_{1} \bar{c}_{-1} \bar{c}_{0} \bar{c}_{1}\right| 0\right\rangle \equiv-i,
$$

and it agrees with textbook conventions [49, to which we adhere in this paper.
} 
The right hand side can be computed by expressing $\left(c_{0}-\bar{c}_{0}\right)$ as a contour integral, mapping the disk to the upper half plane and using the doubling trick. Normalizing the basic ghost correlator in the usual way

$$
\left\langle c\left(z_{1}\right) c\left(z_{2}\right) c\left(z_{3}\right)\right\rangle=z_{12} z_{13} z_{23},
$$

where $z_{i j} \equiv z_{i}-z_{j}$, we find

$$
\left\langle\left(c_{0}-\bar{c}_{0}\right) c \bar{c}(0)\right\rangle_{\text {disk }}=-2,
$$

and the normalization of the ghost boundary state is thus given simply by

$$
\mathcal{N}_{g h}=1 .
$$




\section{Some more lumps}

Here we collect the gauge invariant data of few more lump solutions. All data have been obtained in the $(L, 3 L)$ scheme up to $L=12$.

- Single lump at $R=\sqrt{3}$

This is the same solution of MSZ [35] but in the $(L, 3 L)$ scheme.

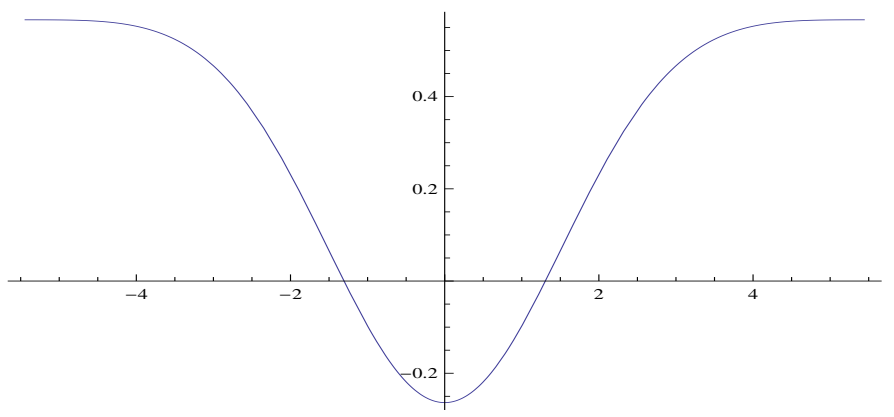

Figure D.1: Open string tachyon profile of the MSZ single-lump solution obtained at $R=\sqrt{3}$ and level $L=(12,36)$.

\begin{tabular}{|c|c|ccccccc|c|}
\hline$L$ & Action & $E_{0}$ & $E_{1}$ & $E_{2}$ & $E_{3}$ & $E_{4}$ & $E_{5}$ & $E_{6}$ & $D$ \\
\hline 1 & 1.32002 & 1.23951 & 0.74368 & - & - & - & - & - & 1.23951 \\
\hline 2 & 1.09428 & 1.09094 & 0.830804 & 1.03277 & - & - & - & - & -1.01353 \\
\hline 3 & 1.06053 & 1.06017 & 0.905713 & 1.08758 & 1.36793 & - & - & - & -1.11078 \\
\hline 4 & 1.03572 & 1.04623 & 0.918393 & 0.931471 & 1.4122 & - & - & - & -0.752479 \\
\hline 5 & 1.02936 & 1.03948 & 0.94075 & 0.933722 & 0.678169 & - & - & - & -0.779229 \\
\hline 6 & 1.02141 & 1.02921 & 0.946315 & 0.995166 & 0.676601 & 2.06251 & - & - & -0.945165 \\
\hline 7 & 1.01868 & 1.02668 & 0.956761 & 0.996584 & 1.11184 & 2.11211 & - & - & -0.959492 \\
\hline 8 & 1.01454 & 1.02301 & 0.959784 & 0.977037 & 1.12839 & -0.327725 & - & - & -0.909528 \\
\hline 9 & 1.01351 & 1.02171 & 0.965702 & 0.976881 & 0.859033 & -0.350675 & 3.66745 & - & -0.913363 \\
\hline 10 & 1.01108 & 1.01787 & 0.967666 & 0.993958 & 0.860958 & 1.98806 & 3.81063 & - & -0.963774 \\
\hline 11 & 1.01052 & 1.01708 & 0.971569 & 0.993933 & 1.04829 & 2.01551 & -4.09339 & - & -0.966875 \\
\hline 12 & 1.00893 & 1.01549 & 0.972933 & 0.98699 & 1.05428 & -0.0353736 & -4.26896 & 8.53484 & -0.945928 \\
\hline Exp. & 1 & 1 & 1 & 1 & 1 & 1 & 1 & 1 & -1 \\
\hline
\end{tabular}


- Single lump at $R=2 \sqrt{3}$

This is a single lump centered at $x=\pi R$. Notice how this reflects into alternating signs for the $E_{n}$ invariants.

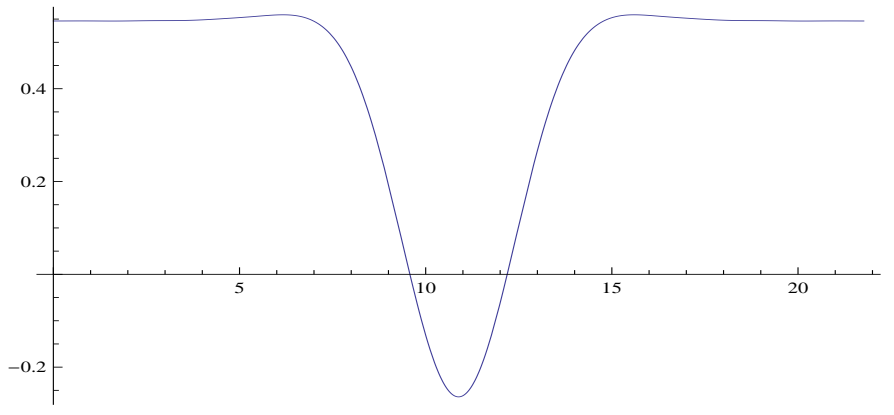

Figure D.2: Open string tachyon profile of a single-lump solution obtained at $R=2 \sqrt{3}$ and level $L=(12,36)$.

\begin{tabular}{|c|c|c|cccccc|}
\hline$L$ & Action & $D$ & $E_{0}$ & $E_{1}$ & $E_{2}$ & $E_{3}$ & $E_{4}$ & $E_{5}$ \\
\hline 1 & 1.84419 & 1.70138 & 1.70138 & -0.72894 & 0.747337 & -0.723963 & - & - \\
\hline 2 & 1.20098 & -0.467964 & 1.30548 & -0.77845 & 0.846539 & -0.91968 & 0.949864 & - \\
\hline 3 & 1.13151 & -0.90771 & 1.25135 & -0.880111 & 0.906433 & -0.928996 & 1.08763 & -1.21329 \\
\hline 4 & 1.05813 & -0.644043 & 1.1658 & -0.893358 & 0.922018 & -0.945655 & 0.92909 & -1.25847 \\
\hline 5 & 1.05079 & -0.677223 & 1.15751 & -0.926427 & 0.941257 & -0.963228 & 0.933319 & -0.854891 \\
\hline 6 & 1.02895 & -0.851623 & 1.11426 & -0.931712 & 0.947518 & -0.970614 & 0.993906 & -0.85989 \\
\hline 7 & 1.02724 & -0.875745 & 1.11142 & -0.945795 & 0.957258 & -0.970967 & 0.997313 & -1.03384 \\
\hline 8 & 1.01773 & -0.84127 & 1.09036 & -0.948533 & 0.960557 & -0.974792 & 0.976872 & -1.04075 \\
\hline 9 & 1.01724 & -0.847968 & 1.08895 & -0.957215 & 0.966076 & -0.979099 & 0.977358 & -0.949906 \\
\hline 10 & 1.01217 & -0.909139 & 1.07312 & -0.958953 & 0.968161 & -0.981546 & 0.993873 & -0.952527 \\
\hline 11 & 1.01204 & -0.915063 & 1.07228 & -0.964312 & 0.971903 & -0.981671 & 0.994434 & -1.00923 \\
\hline 12 & 1.00897 & -0.897553 & 1.06302 & -0.965506 & 0.973333 & -0.983331 & 0.98709 & -1.01181 \\
\hline Exp. & 1 & -1 & 1 & -1 & 1 & -1 & 1 & -1 \\
\hline
\end{tabular}

\begin{tabular}{|c|ccccccc|}
\hline$L$ & $E_{6}$ & $E_{7}$ & $E_{8}$ & $E_{9}$ & $E_{10}$ & $E_{11}$ & $E_{12}$ \\
\hline 1 & - & - & - & - & - & - & - \\
\hline 2 & - & - & - & - & - & - & - \\
\hline 3 & 1.33609 & - & - & - & - & - & - \\
\hline 4 & 1.38348 & - & - & - & - & - & - \\
\hline 5 & 0.676515 & -1.67643 & - & - & - & - & - \\
\hline 6 & 0.677062 & -1.72458 & 2.03206 & - & - & - & - \\
\hline 7 & 1.1127 & -0.327417 & 2.12122 & -2.62668 & - & - & - \\
\hline 8 & 1.12425 & -0.320042 & -0.314551 & -2.71207 & - & - & - \\
\hline 9 & 0.859104 & -1.3691 & -0.352809 & 1.55456 & 3.69837 & - & - \\
\hline 10 & 0.860785 & -1.3843 & 1.97233 & 1.61552 & 3.78564 & - & - \\
\hline 11 & 1.04906 & -0.617564 & 2.01885 & -3.49068 & -4.12322 & -5.50346 & - \\
\hline 12 & 1.05315 & -0.615319 & -0.0261646 & -3.56055 & -4.23092 & -5.60153 & 8.48854 \\
\hline Exp. & 1 & -1 & 1 & -1 & 1 & -1 & 1 \\
\hline
\end{tabular}


- Symmetric double lump at $R=2 \sqrt{3}$ This solution represents two D-branes at distance $a=\frac{1}{2}$, as defined in section 4.2. This double lump solution is just the single lump solution we obtained at $R=\sqrt{3}$, translated by half the period $\pi \sqrt{3}$ and periodically extended to circle of radius $R=2 \sqrt{3}$. This is clearly visible from the invariants, which, up to the alternating signs, are exactly double of those for the single lump at $R=\sqrt{3}$.

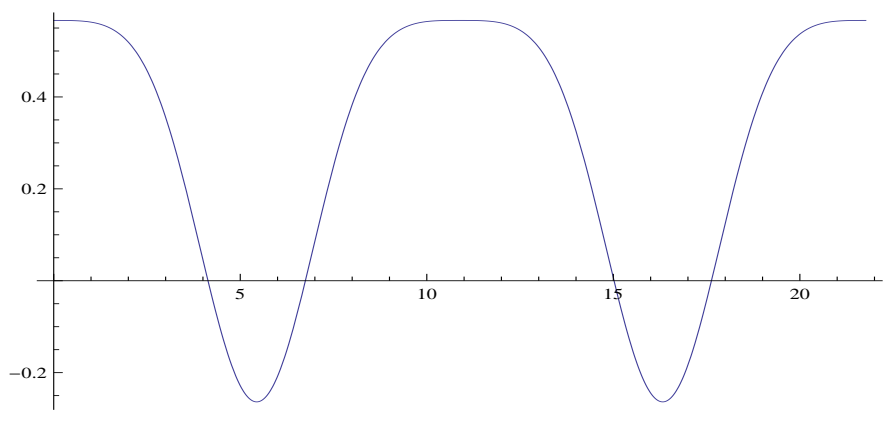

Figure D.3: Open string tachyon profile of a symmetric double-lump solution obtained at $R=2 \sqrt{3}$ and level $L=(12,36)$.

\begin{tabular}{|c|c|c|cccccc|}
\hline$L$ & Action & $D$ & $E_{0}$ & $E_{1}$ & $E_{2}$ & $E_{3}$ & $E_{4}$ & $E_{5}$ \\
\hline 1 & 2.64005 & 2.47902 & 2.47902 & 0 & -1.48736 & - & - & - \\
\hline 2 & 2.18856 & -2.02706 & 2.18187 & 0 & -1.66161 & 0 & 2.06554 & - \\
\hline 3 & 2.12107 & -2.22155 & 2.12035 & 0 & -1.81143 & 0 & 2.17516 & 0 \\
\hline 4 & 2.07144 & -1.50496 & 2.09245 & 0 & -1.83679 & 0 & 1.86294 & 0 \\
\hline 5 & 2.05871 & -1.55846 & 2.07896 & 0 & -1.8815 & 0 & 1.86744 & 0 \\
\hline 6 & 2.04179 & -1.89033 & 2.05843 & 0 & -1.89263 & 0 & 1.99033 & 0 \\
\hline 7 & 2.03736 & -1.91898 & 2.05336 & 0 & -1.91352 & 0 & 1.99317 & 0 \\
\hline 8 & 2.02908 & -1.81906 & 2.04602 & 0 & -1.91957 & 0 & 1.95407 & 0 \\
\hline 9 & 2.02702 & -1.82673 & 2.04341 & 0 & -1.9314 & 0 & 1.95376 & 0 \\
\hline 10 & 2.02216 & -1.92755 & 2.03574 & 0 & -1.93533 & 0 & 1.98792 & 0 \\
\hline 11 & 2.02103 & -1.93375 & 2.03417 & 0 & -1.94314 & 0 & 1.98787 & 0 \\
\hline 12 & 2.01785 & -1.89186 & 2.03098 & 0 & -1.94587 & 0 & 1.97398 & 0 \\
\hline Expected & 2 & -2 & 2 & 0 & -2 & 0 & 2 & 0 \\
\hline
\end{tabular}




\begin{tabular}{|c|ccccccc|}
\hline$L$ & $E_{6}$ & $E_{7}$ & $E_{8}$ & $E_{9}$ & $E_{10}$ & $E_{11}$ & $E_{12}$ \\
\hline 1 & - & - & - & - & - & - & - \\
\hline 2 & - & - & - & - & - & - & - \\
\hline 3 & -2.73586 & - & - & - & - & - & - \\
\hline 4 & -2.82439 & - & - & - & - & - & - \\
\hline 5 & -1.35634 & 0 & - & - & - & - & - \\
\hline 6 & -1.3532 & 0 & 4.12501 & - & - & - & - \\
\hline 7 & -2.22369 & 0 & 4.22422 & 0 & - & - & - \\
\hline 8 & -2.25678 & 0 & -0.655451 & 0 & - & - & - \\
\hline 9 & -1.71807 & 0 & -0.701349 & 0 & -7.3349 & - & - \\
\hline 10 & -1.72192 & 0 & 3.97612 & 0 & -7.62125 & - & - \\
\hline 11 & -2.09657 & 0 & 4.03102 & 0 & 8.18679 & 0 & - \\
\hline 12 & -2.10857 & 0 & -0.0707473 & 0 & 8.53792 & 0 & 17.0697 \\
\hline Expected & -2 & 0 & 2 & 0 & -2 & 0 & 2 \\
\hline
\end{tabular}

\section{References}

[1] E. Witten, "Noncommutative Geometry And String Field Theory," Nucl. Phys. B 268, 253 (1986).

[2] W. Taylor and B. Zwiebach, "D-branes, tachyons, and string field theory," arXiv:hep-th/0311017.

[3] E. Fuchs and M. Kroyter, "Analytical Solutions of Open String Field Theory," arXiv:0807.4722 [hep-th].

[4] A. Sen, "Energy momentum tensor and marginal deformations in open string field theory," JHEP 0408 (2004) 034 [hep-th/0403200].

[5] I. Ellwood, "The Closed string tadpole in open string field theory," JHEP 0808 (2008) 063 arXiv:0804.1131 [hep-th]].

[6] M. Kiermaier, Y. Okawa and B. Zwiebach, "The boundary state from open string fields," arXiv:0810.1737 [hep-th].

[7] T. Kawano, I. Kishimoto and T. Takahashi, "Gauge Invariant Overlaps for Classical Solutions in Open String Field Theory," Nucl. Phys. B 803 (2008) 135 arXiv:0804.1541 [hep-th]].

[8] N. Ishibashi, "The Boundary and Crosscap States in Conformal Field Theories," Mod. Phys. Lett. A 4 (1989) 251.

[9] A. Sen, "Universality of the tachyon potential," JHEP 9912 (1999) 027 hep-th/9911116. 
[10] L. Rastelli and B. Zwiebach, "Tachyon potentials, star products and universality," JHEP 0109 (2001) 038 [hep-th/0006240].

[11] D. Takahashi, "The boundary state for a class of analytic solutions in open string field theory," JHEP 1111 (2011) 054 [arXiv:1110.1443 [hep-th]].

[12] A. Rajaraman and M. Rozali, "D-branes in linear dilaton backgrounds," JHEP 9912 (1999) 005 hep-th/9909017].

[13] M. Gaberdiel, "Boundary conformal field theory and D-branes", Lectures given at the TMR network school on Nonperturbative methods in low dimensional integrable models, Budapest, 15-21 July 2003. http://www.phys.ethz.ch/ mrg/lectures2.pdf

[14] M. Kiermaier and Y. Okawa, "Exact marginality in open string field theory: A General framework," JHEP 0911 (2009) 041 [arXiv:0707.4472 [hep-th]].

[15] E. Fuchs, M. Kroyter and R. Potting, "Marginal deformations in string field theory," JHEP 0709 (2007) 101 arXiv:0704.2222 [hep-th]].

[16] L. Bonora, C. Maccaferri and D. D. Tolla, "Relevant Deformations in Open String Field Theory: a Simple Solution for Lumps," JHEP 1111 (2011) 107 arXiv:1009.4158 [hep-th]].

[17] M. Murata and M. Schnabl, "Multibrane Solutions in Open String Field Theory," JHEP 1207 (2012) 063 [arXiv:1112.0591 [hep-th]].

[18] M. Schnabl, "Comments on marginal deformations in open string field theory," Phys. Lett. B 654 (2007) 194 hep-th/0701248 [HEP-TH]].

[19] M. Kiermaier, Y. Okawa, L. Rastelli and B. Zwiebach, "Analytic solutions for marginal deformations in open string field theory," JHEP 0801 (2008) 028 hep-th/0701249 [HEP-TH]].

[20] T. Erler, "Marginal Solutions for the Superstring," JHEP 0707 (2007) 050 arXiv:0704.0930 [hep-th]].

[21] M. Kiermaier, Y. Okawa and P. Soler, "Solutions from boundary condition changing operators in open string field theory," JHEP 1103 (2011) 122 arXiv:1009.6185 [hepth]].

[22] M. Schnabl, "Analytic solution for tachyon condensation in open string field theory," Adv. Theor. Math. Phys. 10 (2006) 433 arXiv:hep-th/0511286. 
[23] Y. Okawa, "Comments on Schnabl's analytic solution for tachyon condensation in Witten's open string field theory," JHEP 0604 (2006) 055 [arXiv:hep-th/0603159].

[24] T. Erler, "Split string formalism and the closed string vacuum," JHEP 0705 (2007) 083 [arXiv:hep-th/0611200].

[25] T. Erler, "Split string formalism and the closed string vacuum. II," JHEP 0705 (2007) 084 arXiv:hep-th/0612050.

[26] M. Schnabl, "Algebraic solutions in Open String Field Theory - a lightning review," arXiv:1004.4858 [hep-th].

[27] I. Kishimoto, "Comments on gauge invariant overlaps for marginal solutions in open string field theory," Prog. Theor. Phys. 120 (2008) 875 [arXiv:0808.0355 [hep-th]].

[28] T. Noumi and Y. Okawa, "Solutions from boundary condition changing operators in open superstring field theory," JHEP 1112 (2011) 034 [arXiv:1108.5317 [hep-th]].

[29] T. Erler and C. Maccaferri, "The Phantom Term in Open String Field Theory," JHEP 1206 (2012) 084 [arXiv:1201.5122 [hep-th]].

[30] T. Erler and C. Maccaferri, "Connecting Solutions in Open String Field Theory with Singular Gauge Transformations," JHEP 1204 (2012) 107 [arXiv:1201.5119 [hep-th]].

[31] A. Sen, "Tachyon dynamics in open string theory," Int. J. Mod. Phys. A 20 (2005) 5513 [hep-th/0410103].

[32] F. Larsen, A. Naqvi and S. Terashima, "Rolling tachyons and decaying branes," JHEP 0302 (2003) 039 hep-th/0212248.

[33] I. Ellwood, "Rolling to the tachyon vacuum in string field theory," JHEP 0712 (2007) 028 arXiv:0705.0013 [hep-th]].

[34] S. Hellerman and M. Schnabl, "Light-like tachyon condensation in Open String Field Theory," arXiv:0803.1184 [hep-th].

[35] N. Moeller, A. Sen, B. Zwiebach, "D-branes as tachyon lumps in string field theory," JHEP 0008 (2000) 039. hep-th/0005036].

[36] N. Moeller, "Codimension two lump solutions in string field theory and tachyonic theories," hep-th/0008101.

[37] M. Beccaria, "D0-brane tension in string field theory," JHEP 0509 (2005) 021 hep-th/0508090]. 
[38] A. Kurs, "Classical solutions in string field theory," Senior thesis, Princeton University, 2005

[39] M. Kudrna, M. Schnabl, to appear

[40] M. Kudrna, T. Masuda, Y. Okawa, M. Schnabl and K. Yoshida, "Gauge-invariant observables and marginal deformations in open string field theory," arXiv:1207.3335 [hep-th].

[41] T. Erler, "Exotic Universal Solutions in Cubic Superstring Field Theory," JHEP 1104 (2011) 107 arXiv:1009.1865 [hep-th]].

[42] A. Sen and B. Zwiebach, "Large marginal deformations in string field theory," JHEP 0010 (2000) 009 [hep-th/0007153].

[43] J. L. Karczmarek and M. Longton, "SFT on separated D-branes and D-brane translation," arXiv:1203.3805 [hep-th].

[44] H. G. Kausch, "Curiosities at c $=-2$," hep-th/9510149.

[45] D. Gaiotto and L. Rastelli, "A Paradigm of open / closed duality: Liouville D-branes and the Kontsevich model," JHEP 0507 (2005) 053 [hep-th/0312196].

[46] M. Schnabl, "Wedge states in string field theory," JHEP 0301 (2003) 004 hep-th/0201095].

[47] A. LeClair, M. E. Peskin and C. R. Preitschopf, "String Field Theory on the Conformal Plane. 1. Kinematical Principles," Nucl. Phys. B 317 (1989) 411.

[48] B. Zwiebach, "Closed string field theory: Quantum action and the B-V master equation," Nucl. Phys. B 390 (1993) 33 [hep-th/9206084].

[49] J. Polchinski, "String theory. Vol. 1: An introduction to the bosonic string," Cambridge, UK: Univ. Pr. (1998) $402 \mathrm{p}$ 